This item was submitted to Loughborough's Research Repository by the author.

Items in Figshare are protected by copyright, with all rights reserved, unless otherwise indicated.

\title{
VOLCO-X: Numerical simulation of material distribution and voids in extrusion additive manufacturing
}

PLEASE CITE THE PUBLISHED VERSION

https://doi.org/10.1016/j.addma.2021.101900

PUBLISHER

Elsevier

VERSION

AM (Accepted Manuscript)

\section{PUBLISHER STATEMENT}

This paper was accepted for publication in the journal Additive Manufacturing and the definitive published version is available at https://doi.org/10.1016/j.addma.2021.101900

LICENCE

CC BY-NC-ND 4.0

\section{REPOSITORY RECORD}

Macedo, Rafael Quelho de, Rafael Thiago Luiz Ferreira, Andy Gleadall, and lan Ashcroft. 2021. "VOLCO-X: Numerical Simulation of Material Distribution and Voids in Extrusion Additive Manufacturing". Loughborough University. https://hdl.handle.net/2134/14216024.v1. 


\title{
VOLCO-X: numerical simulation of material distribution and voids in extrusion additive manufacturing
}

\author{
Rafael Quelho de Macedo ${ }^{\mathrm{a}}$, Rafael Thiago Luiz Ferreira ${ }^{\mathrm{a}, *}$, Andrew Gleadall $^{\mathrm{b}, \mathrm{c}}$, Ian Ashcroft ${ }^{\mathrm{b}}$ \\ ${ }^{a}$ GPMA, Research Group on Additive Manufacturing, Instituto Tecnólogico de Aeronáutica, 12228-900, São José dos \\ Campos, São Paulo, Brazil. \\ ${ }^{b}$ Centre for Additive Manufacturing, Faculty of Engineering, University of Nottingham, University Park, Nottingham, NG8 \\ $1 B B, U K$ \\ ${ }^{c}$ Wolfson School of Mechanical and Manufacturing Engineering, Loughborough University, Loughborough, Leicestershire, \\ LE11 $3 T U, U K$
}

\begin{abstract}
Parts produced by additive manufacturing have final characteristics (such as mechanical properties and dimensional accuracy) strongly dependent on how material is deposited during production. This study presents a modelling concept called VOLCO-X (VOLume COnserving model - eXtended version), which extends a recently developed simulation technique to be able to accurately simulate deposited structures that were not possible with the previous model. A major advantage of the proposed modelling approach is that it does not require any experimental calibration or fitting. The modelling approach is based on a principle of conservation of volume in a voxelized space, in conjunction with a new deposition modelling concept that re-distributes the deposited material when neighboring filaments are in contact. In addition, an acceleration-dependent extrusion rate correction was implemented in the software to predict changes in the material distribution as function of the printing speed, as well as a mechanism to effectively consider possible asymmetry of deposited filaments. The model is shown to accurately predict the geometry and porosity of specimens manufactured by Fused Filament Fabrication (FFF) with varied printing speeds, distance between filaments and extrusion widths. The numerical results correlated well with validation experiments, being able to capture the transition from triangle to diamond void shapes and to predict defects observed in printed parts. VOLCO-X could simulate printing conditions from fully dense structures to under-extruded structures with gaps. It has potential to aid in the design of functional printed parts by predicting the final dimensions, void shapes and void volume fraction of 3D printed parts, and represents an important step towards enabling the predictive simulation of full-sized parts.
\end{abstract}

Keywords: fused filament fabrication, 3D printing, material distribution, printing speed, VOLCO

\section{Introduction}

In the 2000's, key patents in the field of additive manufacturing (AM) expired, leading to a consistent increase in the development and use of AM technologies [1], specially due to collaborative open source design, such as the Reprap [2]. There is now a wide range of AM applications, with examples in aerospace [3] 5], automotive [6, 7], biomedical [8, pharmacy [9], dentistry [10, architecture [11, food [12], and other

\footnotetext{
* Corresponding author

Email addresses: rquelho@ita.br (Rafael Quelho de Macedo), rthiago@ita.br (Rafael Thiago Luiz Ferreira ), a.gleadall@lboro.ac.uk (Andrew Gleadall), ian.ashcroft@nottingham.ac.uk (Ian Ashcroft)
} 
industries. Fused Filament Fabrication (FFF) [13, also known as FDM ${ }^{\circledR}$ (Fused Deposition Modelling), is an extrusion based technique inside the broader field of Additive Manufacturing (AM) [14, in which the feedstock material is pushed by an extruder into a heated print head, and exits a nozzle as thin lines of material. The process aim is that thin lines are deposited additively, side-by-side and then layer-by-layer, as the nozzle moves in a specific pattern to form the desired part geometry [15, 16].

The manufacturing process starts with a CAD (Computer Aided Design) model of the solid part which is then converted to a .gcode file by software capable of slicing the 3D solid in layers and representing each layer by a combination of deposited lines. In this file, the printing path is described together with the printing parameters, including printing speed, layer thickness and the temperatures of the nozzle and print bed.

Manufacturing of high-value products is possible with FFF due to recent advances in materials $[17+23]$ and technology [23, 24]. In the context of functional parts manufactured by FFF, the physical properties are highly dependent on the material distribution within the printed part, as is also the case for other AM processes. The additive nature of such processes can create material distributions with voids within the microstructure, which affect the mechanical properties of printed parts 25 27. Voids can also generate stress concentrations 25, 28, which can reduce the printed part strength and affect the printed part's fatigue life [29. The volume of the voids and the bonding between filaments affect the impact strength [30] and the structural integrity 31 of printed parts. The size of the voids and the shape of the deposited filaments affect the compressive modulus [32] and ultimate strength [33]. Different configurations of material distribution within printed parts can influence blood and oxygen flow, and the growth of bone cells in biomedical applications [34].

The phenomenon of material distribution within printed parts is complex and is influenced by many variables. One of the first problems investigated in the literature regarding material distribution in FFF printed parts was the sintering process [31, 35, 36]. Sintering in this case can be seen as coalescence of filaments subjected to heat and pressure over time [37. Besides thermal effects, it has been shown that the printing speed [38, nozzle diameter [33], distance between centres of printed filaments [39], layer thickness [33, filament alignment [39] and extrusion flow rate [40] influence the material distribution. \#17 Motor accelerations and printing speeds (both in terms of printing head displacements and filament extrusion rate) also have an important effect on material distribution, e.g. as seen in [41,43] based on computational fluid dynamics models, and are investigated in this work. The importance of taking into account printing accelerations/speeds on the material distribution prediction is clear. For instance, in every printed part, the 
printing speed must be chosen to balance effects on printed part surface quality, manufacturing time and to create adhesion to the print bed (by printing the first layer slowly). Also, the printing speed influences final part dimensions [15] and interlayer material adhesion. Moreover, it can lead to issues such as blocked pores on the surface of scaffold structures or to over-extrusion at the interface between infill and perimeter lines.

Owing to the large number of variables that affect the properties of parts manufactured by FFF, it would be impractical to determine experimentally the influence of all important variables on material distribution. Therefore, numerical methods and simulations capable of predicting material distribution within printed parts are highly desirable. Previous investigations have simulated the extrusion process in FFF in order to predict the distortion caused by the temperature changes during manufacturing [44, but such models are not suitable for large structures due to the computational effort of solving a coupled thermo-mechanical problem. The bond length between parallel filaments was estimated in [45, however details of the geometry between deposited filaments was not captured by this model. In [46], a fluid-dynamics analysis was employed to simulate the extrusion flow, but the computational demand limited this model to 2D; in [47] the geometry of lattice structures was estimated using a stochastic model to predict the diameter variation of the deposited filament along its length, however this model does not consider the influence of printing parameters on the material distribution; other multi-physics simulations were reviewed in $[32,41,43$. However, with all these modelling approaches, a broad study of large structures was not possible due to computational demands.

The software VOLCO 32 circumvented the complexity inherent in explicit modelling of the FFF process by applying a volume conserving methodology to predict the material distribution within printed parts. Advantages of this approach include that it does not require experimental calibration, it has low computational demands and it is able to predict the material distribution with good precision without considering thermal effects. It was demonstrated that VOLCO is able to capture geometric details of printed parts considering several parameters, such as: layer thickness, extrusion flow and raster angle.

Although VOLCO is able to predict the material distribution for many different scenarios, it does not have the ability to consider asymmetry resulting from printing neighboring filaments, as illustrated by Fig 1 (b) from experiments in [39. This asymmetry results from the filament being deposited with one side constrained (by the previously deposited filament) and one side unconstrained (open to the air). Furthermore, important effects of the printing speed and acceleration were not considered in VOLCO.

The present work introduces a new version of the software VOLCO, called VOLCO-X, where a new deposition modelling method is proposed in order to predict changes in material distribution when neighboring filaments are in contact. For this purpose, a new mathematical formulation is derived to take into 


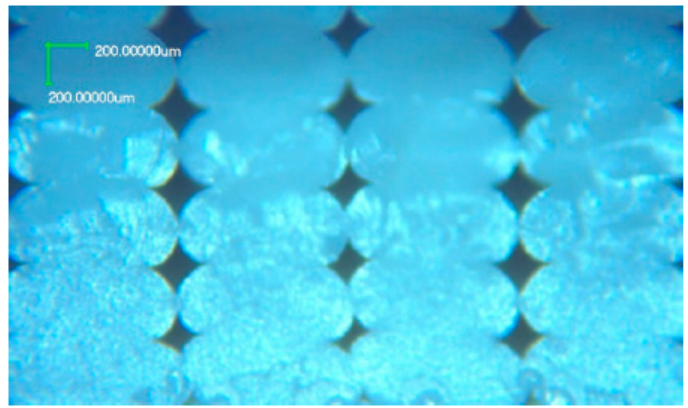

(a)

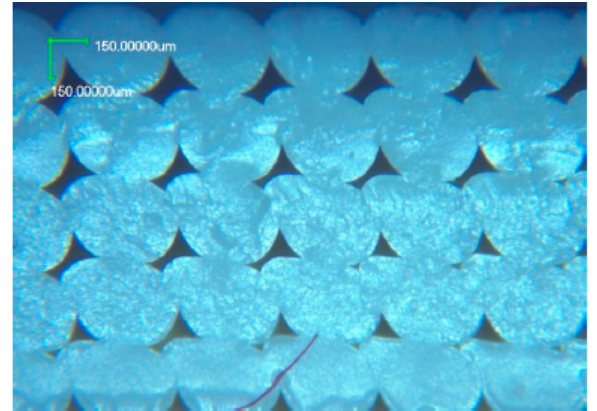

(b)

Figure 1: Microstructure changes according to the distance between centre of deposited filaments: (a) bigger distance between filaments; (b) smaller distance between filaments - Source: 39].

account the contact between deposited filaments during the material deposition process, whilst still considering volume conservation of material (as originally adopted in VOLCO). The interaction between filaments in contact is modelled inspired on experimental observations which are described further in the manuscript.

VOLCO-X also includes an acceleration-dependent extrusion rate modelling method to simulate changes in material distribution as a function of the printing speed. The new computational modelling methods introduced are a significant improvement on the original VOLCO. With VOLCO-X, the material distribution, final dimensions and void shapes within solid layers (100\% infill) can be predicted more accurately. Additionally, the material distribution for non-solid layers (scaffold, honeycombs, triangular and many other infill patterns) can still be predicted with the original VOLCO modelling features. Unlike machine learning (please see reviews in [48, 49]), or purely experimental research, VOLCO-X is able to directly simulate the effect of the print path and printing parameters on the printed microstructure and generates a 3D model of that microstructure.

This paper contributes in the area of additive manufacturing, proposing a simple and computationally efficient simulation to accurately predict the final geometry and material distribution within printed parts. Moreover, VOLCO-X has an important potential to inform printing path planning strategies and predict part faults. There is no other model in the literature able to predict, at a lower computational cost, the material distribution influenced by the printing speed nor able to consider the interaction between neighboring filaments on the prediction of material distribution.

After this introduction, Section 2 briefly presents the original concepts and ideas of VOLCO. The influence of the printing speed on the deposited volume is then investigated according to a conservation of mass procedure in Section 3. The new VOLCO-X procedure for changes in material distribution when the distance between filaments is varied is described in Section 4. The general computational implementation 
procedure is discussed in Section 5. In Section 6, the methodology to validate the numerical model is presented, and the experimental and simulation results are shown in Section 7 In Section 8 , applications and possible further developments are mentioned. Conclusions regarding the numerical model proposed in this work are presented in Section 9

\section{VOLCO original concept}

The VOLCO model is described in 32 but a brief overview is given here. First, a 3D voxel space as depicted in Fig 2(a) is created and, initially, each voxel has value equal to 0 , which means that there is no material in the voxel. The gcode file with the 3D printing build instructions of the part (material distribution) is then imported. The .gcode of the printed part is processed and the length $\left(L_{i}\right)$ and total volume $\left(\Omega_{i}\right)$ of each deposited filament $i$ are defined. The deposition of filament $i$ is then discretized in $N$ simulation steps as shown in Fig 2(b). The original version of VOLCO considers a constant material flow through the nozzle, i.e. there is no influence of the printing speed on the material distribution. Because of this, in each simulation step $n$, a volume $\Omega_{i} / N$ is added to the voxel space.

Voxel Space

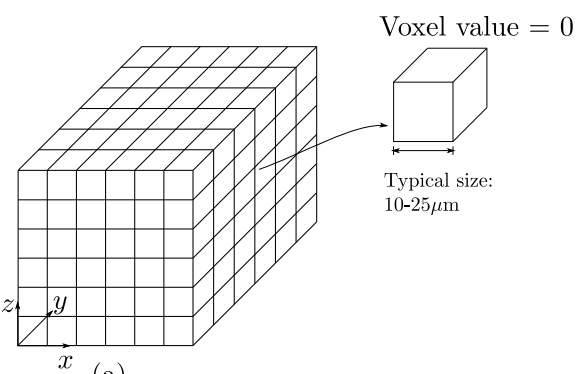

(a)
Filament i

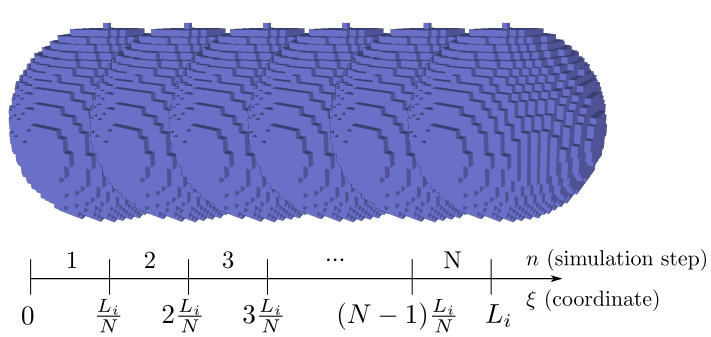

(b)

Figure 2: (a) Voxel space used in VOLCO; (b) deposition of filament $i$ in $N$ simulation steps.

For the deposition of filament $i$, when simulating the step $n$, a sphere with initial radius $r_{s}=0$ is inserted into the $3 \mathrm{D}$ voxel space at the position $\xi=n \frac{L_{i}}{N}$ and $r_{s}$ increases until the volume $\Omega_{i} / N$ is added to the voxel space, as in Fig 3 . When $\Omega_{i} / N$ is reached, the voxels inside the sphere have value equal to 1. Fig 22(b) illustrates the deposition simulation of filament $i$. This process continues until the entire printed part is deposited into the voxel space.

The sphere radius $r_{s}$ that adds a volume $\Omega_{i}$ to the voxel space can be calculated directly if the current deposited sphere is not in contact with any other deposited filament or print bed. In this scenario, the radius is equal to $r_{s}=\sqrt[3]{3 \Omega_{i} / N} / 4 \pi$ (which is the radius of a sphere with volume $\Omega_{i} / N$, and equal to the nominal deposited filament diameter). However, most often, the current sphere is in contact with another 
sphere or the print bed. In this situation, a fraction of the volume of the current sphere will be overlapped by the adjacent one, as seen in Fig.3. In this scenario, an incremental radius $\Delta r_{s}$ should be added to $r_{s}$ in order to deposit the desired volume $\Omega_{i} / N$.
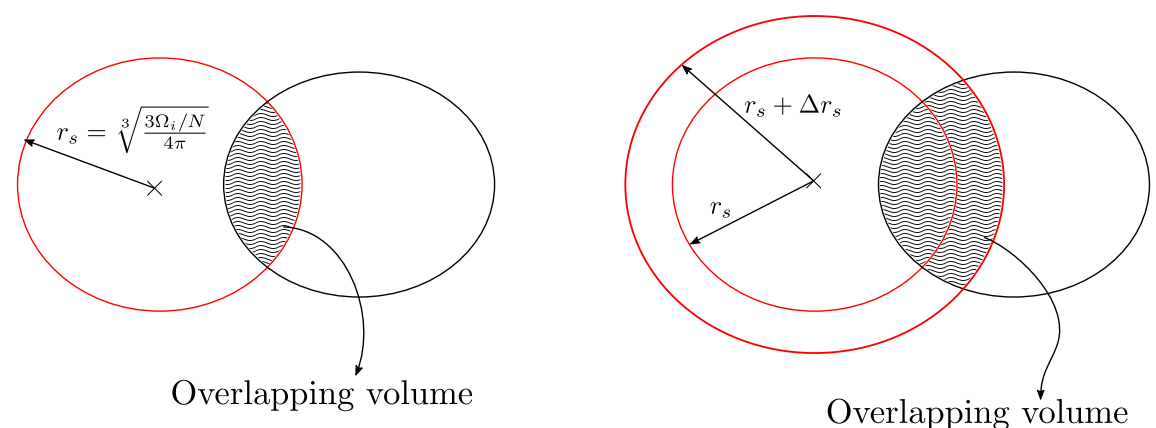

Figure 3: Overlapping volume between spheres lead to higher sphere radius in order to deposit the volume $\Omega_{i} / N$.

The following assumptions were considered and discussed in VOLCO and are also implemented in this work: the material cannot be deposited below the print bed; gravity is not considered; material does not flow after being deposited into the voxel space; there is no contraction or expansion due to temperature changes.

\section{Printing speed and deposited volume per filament}

It is shown in this section, how the printing speed affects the deposited volume of a single filament. The formulation depicted below was implemented in VOLCO-X to predict the material distribution as function of the printing speed. Consistent material flow through the nozzle is assumed.

\subsection{Conservation of mass}

For a given control volume $\Omega$ fixed in space, if no mass is being accumulated inside $\Omega$ and the density $\rho$ of the flow material is constant, the relationship of Eq.(1) describes the mass flow of the control volume.

$$
\dot{m}_{\text {in }}=\dot{m}_{\text {out }} \rightarrow \rho \dot{v}_{\text {in }}=\rho \dot{v}_{\text {out }} \rightarrow(V A)_{\text {in }}=(V A)_{\text {out }} .
$$

In Eq.(1), $\dot{m}$ is the mass flow rate, $\dot{v}$ is the volume flow rate, $A$ is the normal area to the material flow and $V$ is the speed at the same normal direction.

Figure 4 schematically shows the printing process for a single filament. The filament has initial diameter $D_{0}$ and is pushed towards the nozzle by the extruder motor with speed $V_{e}$. The filament exits the nozzle 


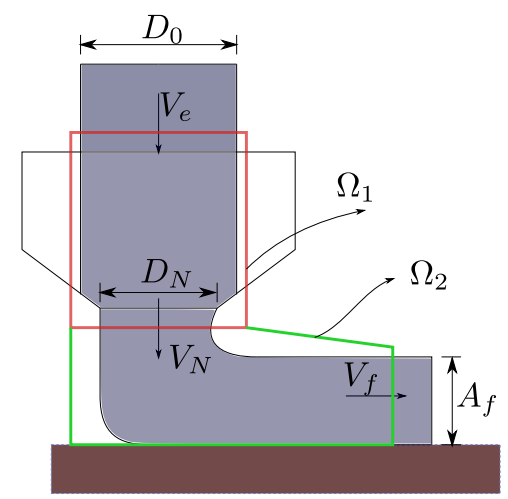

Figure 4: Conservation of mass of a single printed filament.

with diameter $D_{N}$, which is the diameter of the nozzle, with speed $V_{N}$. Then, the material is deposited into the print bed and turns $90^{\circ}$. The printed filament on the bed has area $A_{f}$ and speed $V_{f}$.

In the same figure two control volumes can be seen: $\Omega_{1}$ and $\Omega_{2}$. Assuming that the filament has a circular shape before entering the nozzle and the speeds $V_{e}, V_{N}$ and $V_{f}$ are normal average speeds through cross sections, applying the conservation of mass in $\Omega_{1}$ and $\Omega_{2}$, it is possible to show that the area of the filament deposited onto the print bed is given by Eq.(2), where $t$ is time.

$$
A_{f}(t)=\pi \frac{D_{0}^{2}}{4} \frac{V_{e}(t)}{V_{f}(t)}
$$

\subsection{Calculating the deposited volume per filament}

It can be seen in Eq. 22 that the cross sectional area of the printed filament can vary in time if the ratio $V_{e}(t) / V_{f}(t)$ is not constant. In a real $3 \mathrm{D}$ printing process, that ratio might not be constant, due to different nozzle and extruder speed profiles, as explained below.

The .gcode instruction defines the nozzle translation speed $\bar{V}_{f}$, the length of printed filament $L$ and the length of bulk filament that must enter the extruder $E$. (Considering $D_{0}$, the initial filament diameter, $E$ implies in a nominal volume of $\pi \frac{D_{0}^{2}}{4} E$ of material to be extruded). The firmware of the $3 \mathrm{D}$ printer determines the speed profile of the nozzle and extruder (as depicted in Fig 5), and takes into account a nominal speed change threshold - which is commonly known as the Jerk speed. The print head and extruder speed change thresholds are called here as $V_{t}$ and $V_{t e}$, respectively. These depend on each motor configuration. For speeds below this parameter, it is assumed that the motor is able to achieve the desired speed instantly, because the motor has assumed infinite acceleration for speeds below this parameter. This is of course theoretical, and in practice is a very high acceleration. However, for speeds above this parameter, finite acceleration is 
defined.

Nozzle

Extruder

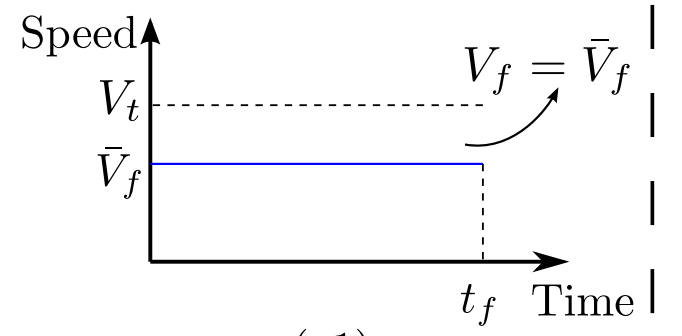

(a1)

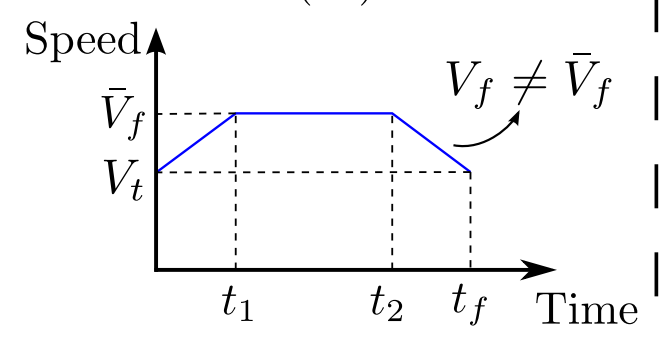

(a2)

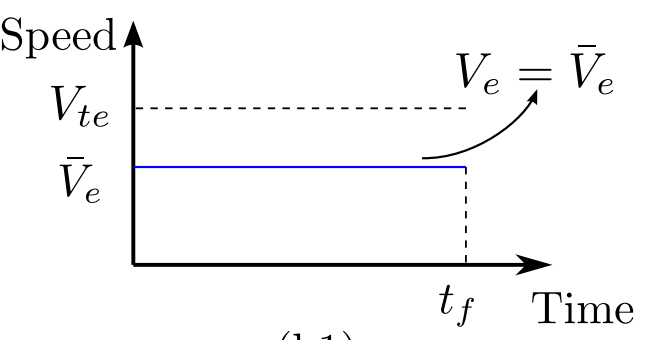

(b1)

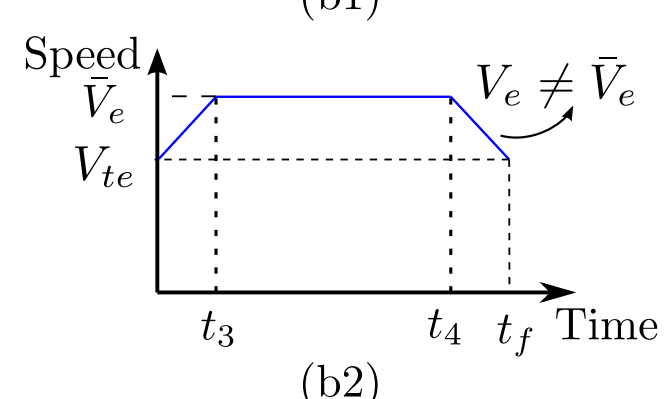

(b2)

Figure 5: Speed profiles in time domain: (a1) and (a2) are the nozzle speeds when $\bar{V}_{f} \leq V_{t}$ and $\bar{V}_{f}>V_{t}$, respectively; (b1) and (b2) are the extruder speeds when $\bar{V}_{e} \leq V_{t e}$ and $\bar{V}_{e}>V_{t e}$, respectively.

It can be seen in Fig 5 (a1) that if $\bar{V}_{f} \leq V_{t}$ ( $V_{t}$ being the speed change threshold of the nozzle), the speed is constant, however, if $\bar{V}_{f}>V_{t}$ the nozzle accelerates from $V_{t}$, reaching the desired speed $\bar{V}_{f}$ and then decelerates to $V_{t}$, as depicted in Fig 5 (a2). Considering the nozzle acceleration $a_{f}$, the time $t_{f}$ required to print the filament can be calculated from Eq.(3):

$$
\begin{array}{r}
t_{f}=\frac{L}{\bar{V}_{f}}, \text { if } \bar{V}_{f} \leq V_{t}, \\
t_{f}=\frac{L}{\bar{V}_{f}}+\frac{\bar{V}_{f}}{a_{f}}-2 \frac{V_{t}}{a_{f}}+\frac{V_{t}^{2}}{\bar{V}_{f} a_{f}}, \text { if } \bar{V}_{f}>V_{t} .
\end{array}
$$

The extruder speed $V_{e}$ is not a printing parameter set in the .gcode, however, it is calculated by the printing machine firmware, using the distance $L$, the total time $t_{f}$ to print the filament, the extruder acceleration $a_{e}$ and the speed change threshold $V_{t e}$ of the extruder axis.

At the moment the nozzle has travelled a distance $L$, the extruder axis will have travelled a distance E. First, it is calculated whether the extruder can print the filament with constant speed as calculated in Eq. (4).

$$
\bar{V}_{e}=V_{e}=E / t_{f}
$$


If $\bar{V}_{e} \leq V_{t e}$, then the extruder speed is $\bar{V}_{e}=E / t_{f}$ and the speed is constant, as depicted in Fig $5(\mathrm{~b} 1)$. However, if $\bar{V}_{e}>V_{t e}$, then the extruder speed is not constant, as shown in Fig 5 (b2). The area under the curve of Fig 5 (b2) must be equal to $E$, so it follows that the extruder speed $\bar{V}_{e}$ is calculated by solving Eq.(5). It is important to note that Eq.(5) has two solutions, and the feasible solution is the one that yields $t_{4}-t_{3}>0$ (this time difference is calculated from Eq. 6 ) $)$.

$$
\begin{array}{r}
\frac{\bar{V}_{e}^{2}}{a_{e}}+\bar{V}_{e}\left[-t_{f}-2 \frac{V_{t e}}{a_{e}}\right]+\left[\frac{V_{t e}^{2}}{a_{e}}+E\right]=0 \\
t_{4}-t_{3}=t_{f}-2 \frac{\bar{V}_{e}}{a_{e}}+2 \frac{V_{t e}}{a_{e}} .
\end{array}
$$

Once $V_{e}$ is calculated, the volume deposited onto the print bed in time can be calculated using Eq.(7).

$$
\Omega_{\mathrm{dep}}(t)=\int_{0}^{t} \pi \frac{D_{0}^{2}}{4} V_{e}(t) d t
$$

Finally, during the material deposition simulated in VOLCO-X, the position $x_{n}(t)$ (being $x_{n}=0$ and $x_{n}=L$ the initial and final positions) of the nozzle is calculated using Eq.(8) and based on this information, the volume of material that should be deposited in the voxel space is calculated by Eq. (9).

$$
\begin{array}{r}
f=x_{n}(t)=\int_{0}^{t} V_{f}(t) d t \\
\Omega_{\mathrm{dep}}\left(t=f^{-1}\left(x_{n}\right)\right)=\int_{0}^{t} \pi \frac{D_{0}^{2}}{4} V_{e}(t) d t .
\end{array}
$$

\section{Contact between filaments and material distribution}

In this section, the new modelling approach implemented in VOLCO-X to predict changes in material distribution when neighboring filaments are in contact is described. In this modelling approach, it is assumed that the volume is conserved during the printing process, as originally assumed in VOLCO.

The new modelling approach is inspired by experimental observations. It can be seen in Fig 1 that the triangle and diamond-like void shapes observed from experiments depend on the distance between the centres of deposited filaments. The reason for this is explained in Fig,6. In Fig 6(a), the distance between the centres of deposited filaments is equal to the filament road width $r$ (which is the width the filament would exhibit if it were not partially blocked by any other filament) and the filament already deposited does not block any material from being deposited. In this case, the diamond void shape is predicted. However, in Fig 6 (b), when the distance between the centres of deposited filaments is smaller than the road width, the 
previously printed filament partially blocks the new filament from being deposited onto the layer below and, because of this, part of the new filament is deposited on top of the old filament, generating the triangle-like void shape.
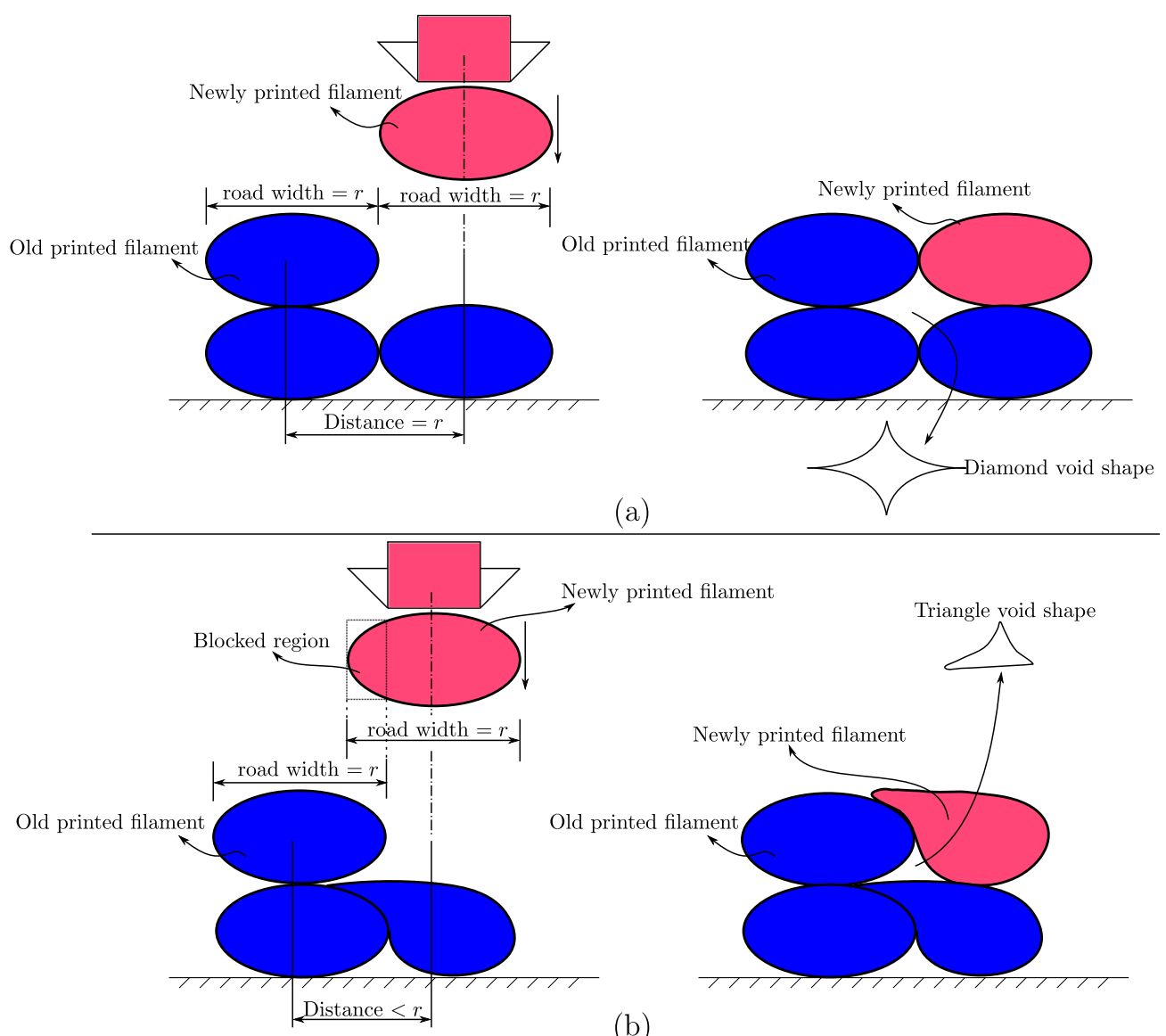

(b)

Figure 6: Possible cases of material distribution considering the distance between the centre of filaments and road width: (a) distance between filaments $=r$; (b) material is blocked by previously printed filament since the distance between filaments $<r$.

In the original formulation of VOLCO, an uniform material growth for each sphere deposited in the voxel space was assumed. In this manner, original VOLCO cannot account for asymmetric changes in material distribution when neighboring filaments are in contact. Therefore, only diamond void shapes can be predicted with VOLCO, as seen in Fig.7(a). In VOLCO-X, a new deposition methodology is developed in order to consider the influence of the contact between filaments in the material distribution. With VOLCO-X, triangle-like void shapes can be predicted, as seen in Fig/7(b).

The new approach to modelling deposition to account for the effect of contact between filaments in the material distribution is described below. It is important to mention that, in VOLCO-X, cylinders are deposited into the 3D voxel space (as in Fig, 8 (a)) rather than the spheres modelled in VOLCO as this is 

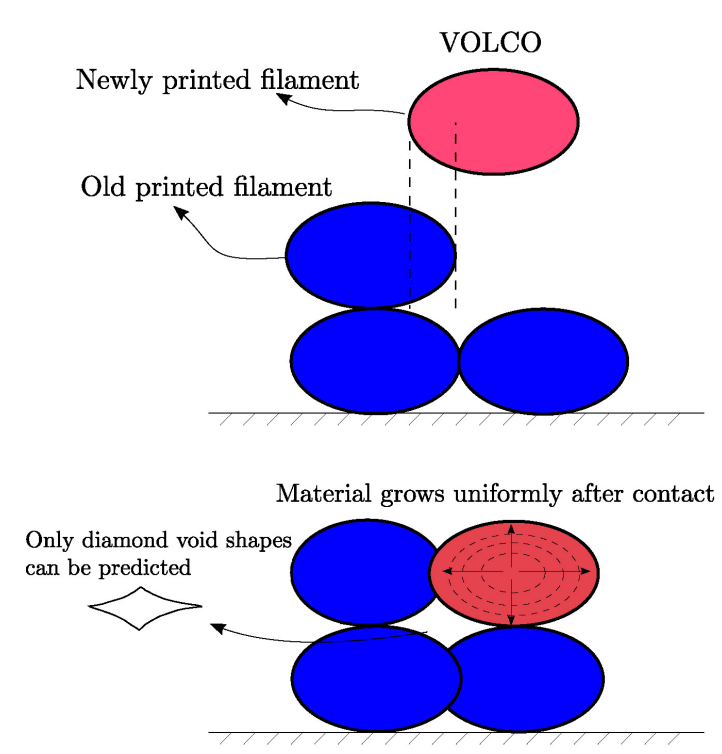

(a)
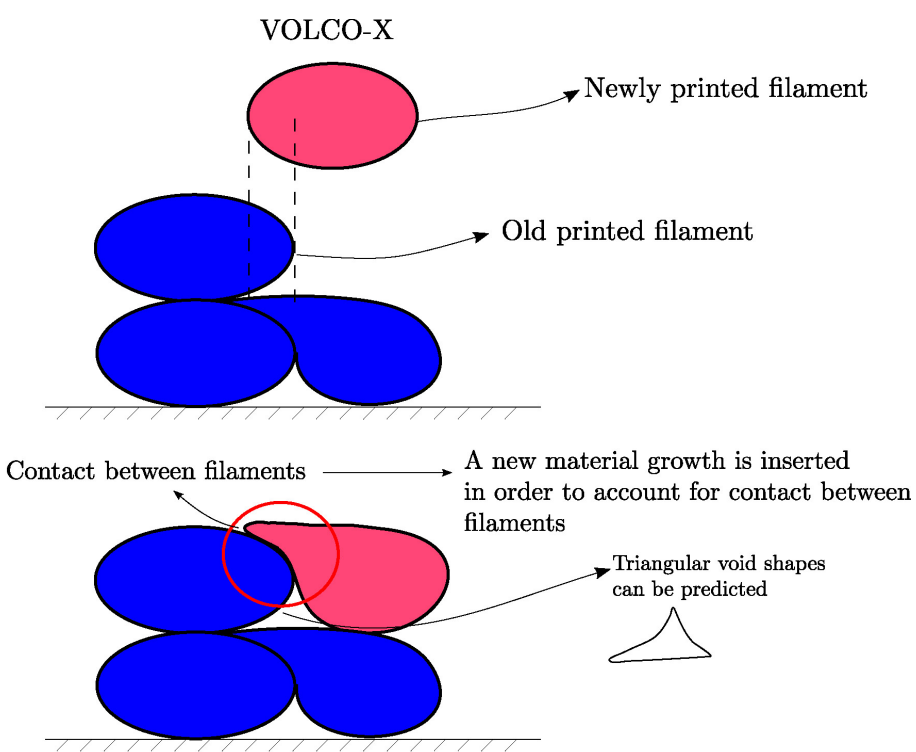

(b)

Figure 7: VOLCO and VOLCO-X capabilities: (a) VOLCO is not able to predict triangle voids because does not account for the contact between filaments; (b) VOLCO-X is able to predict triangle void shapes, since the contact between filaments is modelled.

more computationally efficient.

\subsection{Growth profile before contact of cylinders}

Each printed filament is divided into $N$ cylinders, which is equivalent to $N$ simulation steps per filament. If the filament $i$ is being deposited and has length $L$, each cylinder has length $L_{c}=L / N$. If the current simulation step is $n$, the cross section of the cylinder must increase until the total volume of the printed part reaches the target volume $\Omega_{\text {target }}$, which can be calculated using Eq. 10.

$$
\Omega_{\text {target }}=\Omega_{i-1}+\Omega_{\text {dep }}\left(t=f^{-1}(n L / N)\right) .
$$

In Eq. 10), $\Omega_{i-1}$ is the total volume of the structure calculated after the filament $i-1$ has been deposited and $\Omega_{\mathrm{dep}}\left(t=f^{-1}(n L / N)\right)$ is the volume that is added to the structure for filament $i$ after simulation step $n$, which is calculated from Eq. 99 .

Figures 8(a) and (b) show the coordinate system used in the deposition of cylinders in VOLCO-X and the cross section of each deposited cylinder, respectively. If the distance between the nozzle and the layer below is $H$, the ideal cross section of the filament is a circle with area $A=\pi \frac{H^{2}}{4}$, i.e., it has a geometry that is not deformed by the nozzle nor the layer below. If $A_{f}>A\left(A_{f}\right.$ being the actual area of the filament calculated from Eq.(2) ) and the centre of the cylinder $\left(z_{0}\right)$ is located a distance $H / 2$ from the layer below, 


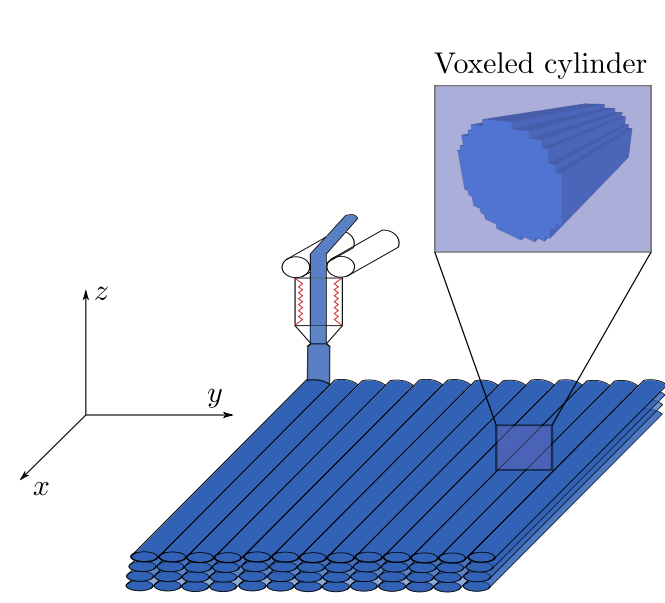

(a)

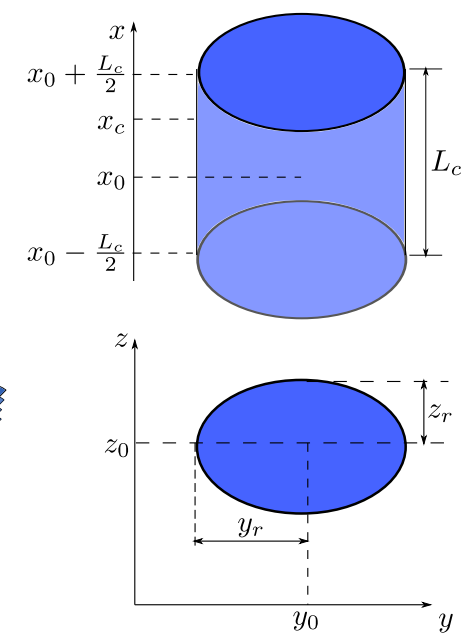

(b)

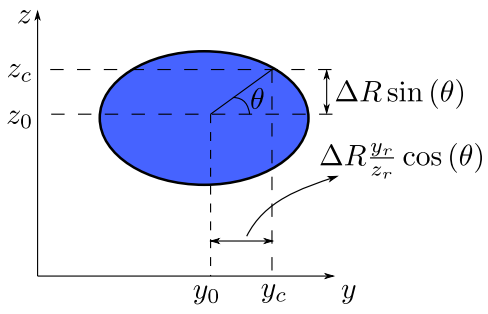

(c)

Figure 8: Deposition of cylinders in the 3D voxel space: (a) coordinate system and example of voxeled cylinder; (b) cross section of a cylinder; (c) points of the cylinder when its cross section is increasing.

the cylinder must have an elliptical shape instead of circular, with a width greater than the height. In this scenario, the ratio $y_{r} / z_{r}$ of the semi-radii (see Fig $8(\mathrm{~b})$ ) is calculated from Eq. (11).

$$
\frac{y_{r}}{z_{r}}=\frac{A_{f}}{A} \rightarrow \frac{y_{r}}{z_{r}}=\frac{4 A_{f}}{\pi H^{2}} .
$$

However, the centre of the cylinder is not always located a distance $H / 2$ from the layer below. In VOLCO-X, $z_{0}$ is the vertical coordinate of a cylinder center and it is located a distance $H-D_{N} / 2$ from the layer below. The horizontal coordinate of a cylinder $y_{0}$ is always at half the prescribed distance between centres of deposited filaments. Both coordinates are seen in Fig 8 (b). The cylinder will have an elliptical shape with $y_{r} / z_{r}$ described by Eq.(11), only if $H=D_{N}$. If the centre of the cylinder is located closer to the layer below than to the nozzle (i.e. when $H<D_{N}$ ), the layer below deforms the cylinder and the shape of the ellipse will have a slight different ratio than $y_{r} / z_{r}$.

In order to account for the previous scenarios of cylinder shape, the cylinder inserted in the 3D voxel space must increase its cross section following the ratio $y_{r} / z_{r}$. Considering the angle $\theta$ (positive in the counter-clockwise direction) and the centre of the cylinder $\left(x_{0}, y_{0}, z_{0}\right)$, the points $\left(x_{c}, y_{c}, z_{c}\right)$ of the cylinder are given by Eq.(12). The angle $\theta$ and the points $\left(x_{c}, y_{c}, z_{c}\right)$ of the cylinder calculated as a function of $\Delta R$ 
can be seen in Fig $8(\mathrm{c})$.

$$
\begin{gathered}
\\
\left\{\begin{array}{c}
x_{c}=x, \\
y_{c}=y_{0}+\Delta R \frac{y_{r}}{z_{r}} \cos \theta, \\
z_{c}=z_{0}+\Delta R \sin \theta,
\end{array}\right. \\
\text { for } \theta \in\left[0,2 \pi\left[, \quad x \in\left[x_{0}-L_{c} / 2, x_{0}+L_{c} / 2[.\right.\right.\right.
\end{gathered}
$$

In Eq. $[12), \Delta R(\Delta R>0)$ is the scalar that causes the increase of the cross section of the cylinder. Then, at the simulation step $n$, the total volume of the structure $\Omega_{\text {Total }}^{n}$ depends on the value of $\Delta R$ $\left(\Omega_{\text {Total }}^{n}=\Omega_{\text {Total }}^{n}(\Delta R)\right)$. When printing filament $i$, the value $\Delta R$ is obtained by solving Eq. 13 .

$$
\begin{array}{r}
\Omega_{\text {target }}-\Omega_{\text {Total }}^{n}(\Delta R)=0 \rightarrow \\
\Omega_{i-1}+\Omega_{\text {dep }}\left(t=f^{-1}(n L / N)\right)-\Omega_{\text {Total }}^{n}(\Delta R)=0 .
\end{array}
$$

\subsection{Growth profile when two cylinders are in contact}

The deposition pattern of Eq. 12 governs the simulation until the moment when the current cylinder is in contact to the adjacent cylinder and the layer below, as in Fig 9 (a). This consideration comes from the fact that if the current filament touches the adjacent cylinder and not the layer below, there is room for more material to be deposited below the current cylinder, as in Fig $9(\mathrm{~b})$; and if the cylinder touches the layer below, but not the adjacent cylinder, the material deposition of the current filament is not blocked by the adjacent filament, as in Fig 9 (c).

During the simulation, if the current cylinder contacts the adjacent cylinder and layer below, but the target volume $\Omega_{\text {target }}$ has not been reached $\left(\Omega_{\text {target }}>\Omega_{\text {Total }}^{n}\right.$ ), a new formulation for the cylinder growth must be used in order to incorporate the phenomenon of old filaments blocking portions of newly printed filaments from being deposited, as explained in Fig 6. In VOLCO-X, two cylinders are in intersection when at least one point of the current and adjacent cylinders have the same spatial coordinates, as depicted in Fig 10 (a). The same is defined for the contact between the current cylinder and the layer below.

At the moment of intersection, two angles $-\theta_{1}$ and $\theta_{1}+\pi$ are defined by the contact between the current cylinder and the layer below, as shown in Fig.10(b). Using Eq. 12 , it is possible to calculate the angle $\theta_{1}$ from Eq. (14). In this equation, $z_{1}$ is the distance between the centre of the current cylinder and the layer 


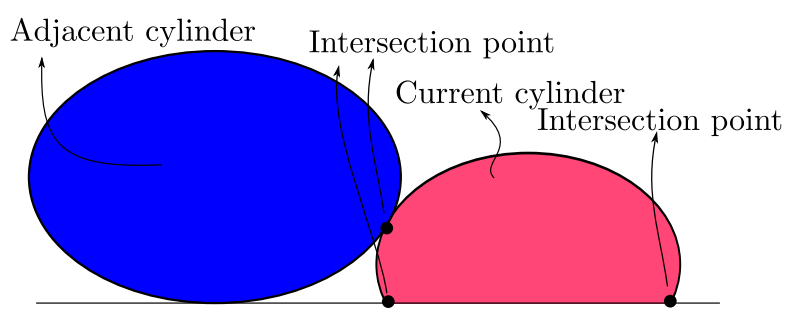

(a)

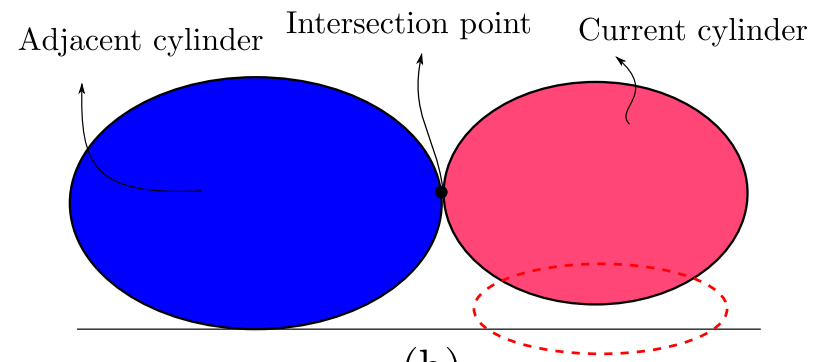

(b)

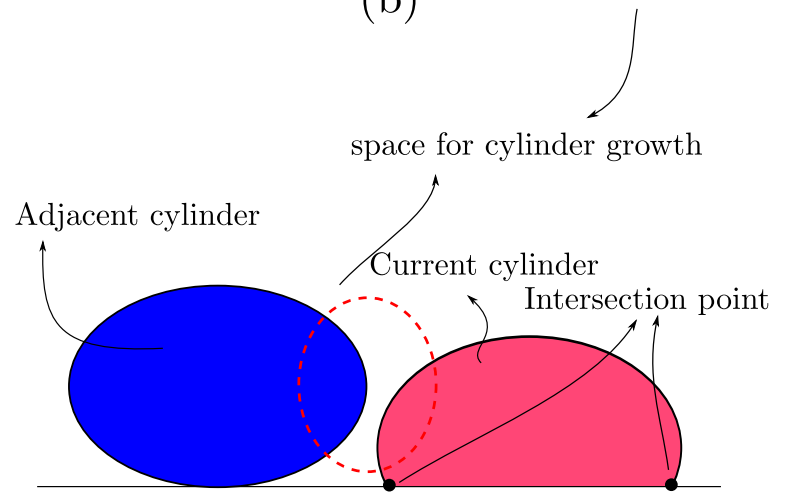

(c)

Figure 9: (a) Cylinder in contact to the adjacent cylinder and layer below; (b) cylinder in contact only with the adjacent cylinder; (c) cylinder in contact only with the layer below.

below and $\Delta \bar{R}$ is the increment in Eq. 12 that caused the intersection of the cylinders.

$$
\theta_{1}=\sin ^{-1}\left(\frac{z_{1}}{\Delta \bar{R}}\right) .
$$

In addition, when the adjacent cylinder blocks new material from being deposited onto the layer below, more material will be deposited on top of the current cylinder and less material will be deposited at the lower portion (see Fig $10(a)$ ) of the current cylinder, as depicted in Fig 6(b). This assumption is incorporated by forcing the distance between the centre of the current cylinder $(O)$ and the outer surface of the cylinder to remain unchanged at angles $-\theta_{1}$ and $\theta_{1}+\pi$. At these angles, the distance that remains unchanged is $R_{b}$ (see Fig $10(\mathrm{~b})$ ), as calculated from Eq. (15). In this equation, $y_{c}\left(-\theta_{1}\right)$ and $z_{c}\left(-\theta_{1}\right)$ are the points of the 

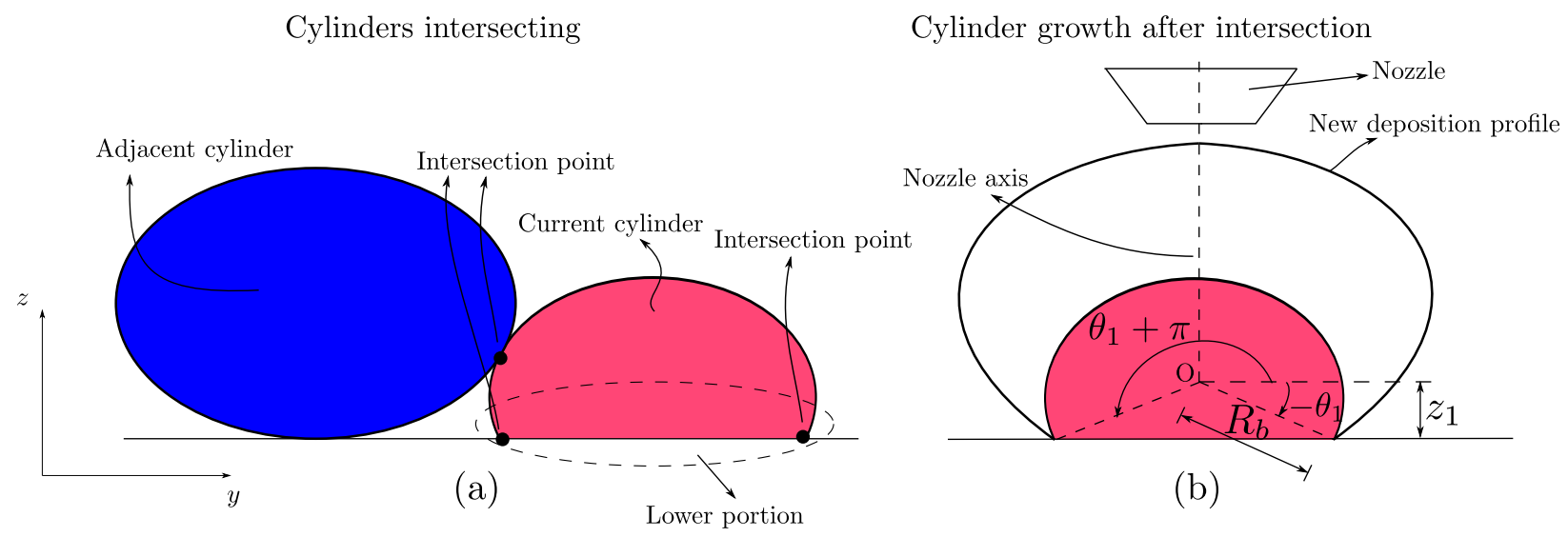

Figure 10: (a) Definition of when two cylinders are in intersection; (b) growth profile of the cylinder after intersection.

outer surface of the current cylinder at $-\theta_{1}$, which are calculated from Eq. 12 .

$$
R_{b}=\left[\left(y_{c}\left(-\theta_{1}\right)-y_{0}\right)^{2}+\left(z_{c}\left(-\theta_{1}\right)-z_{0}\right)^{2}\right]^{\frac{1}{2}}=\left[\left(\Delta \bar{R} \frac{y_{r}}{z_{r}} \cos \theta_{1}\right)^{2}+\left(\Delta \bar{R} \sin \theta_{1}\right)^{2}\right]^{\frac{1}{2}}
$$

It is also considered that, after the intersection, the greatest height of the current cylinder is found at the nozzle axis, as shown in Fig 10 (b), i.e., at $\theta=\pi / 2$. This assumption was inserted in the model in order to consider the simplest deposition profile after the intersection, but respecting the boundary condition at $-\theta_{1}$ and $\theta_{1}+\pi$.

To achieve the above considerations, the periodic function $g(\theta)$, given by Eq.[16], was used to model the deposition profile depicted in Fig 10 (b). It is simple to verify that $g\left(-\theta_{1}\right)=g\left(\theta_{1}+\pi\right)=R_{b}$ (respecting the considerations at $-\theta_{1}$ and $\left.\theta_{1}+\pi\right)$. The symmetry of $g(\theta)$ along the symmetry axis, i.e., along $\theta=\pi / 2$, guarantees that the highest value of this function is found at $\theta=\pi / 2$. In this equation, $C$ is a constant that can change the shape of the function.

$$
g(\theta)=R_{b}+C \sin \left(\frac{\pi\left(\theta+\theta_{1}\right)}{2 \theta_{1}+\pi}\right)
$$

Finally, the new deposition profile can be calculated from Eq. (17) by inserting Eq. $(16)$ in Eq. $(12)$. In this equation, $C_{y}$ and $C_{z}$ are positive scalars that increment the cylinder shape in $y$ and $z$ directions, respectively, in order to achieve the required total deposition volume. It is important to mention that no experimental 
calibration is required to obtain these two constants.

$$
\begin{gathered}
x_{c}=x, \\
\left\{\begin{array}{c}
y_{c}=y_{0}+\left(R_{b}+C_{y} \sin \left(\frac{\pi\left(\theta+\theta_{1}\right)}{2 \theta_{1}+\pi}\right)\right) \cos \theta, \\
z_{c}=z_{0}+\left(R_{b}+C_{z} \sin \left(\frac{\pi\left(\theta+\theta_{1}\right)}{2 \theta_{1}+\pi}\right)\right) \sin \theta,
\end{array}\right. \\
\text { for } \theta \in\left[-\theta_{1}, \theta_{1}+\pi\left[, \quad x \in\left[x_{0}-L_{c} / 2, x_{0}+L_{c} / 2[.\right.\right.\right.
\end{gathered}
$$

It is assumed, in the model, that the region to the left of the centre of the cylinder (see Fig 11(a)) has its geometry affected by the adjacent cylinder, since there is contact between the left region and the adjacent cylinder. This contact is incorporated in the model by splitting Eq. 17] in two: one equation for the left region and one for the right region, each region has its own scalars $C_{y}$ and $C_{z}$. The scalars of the right region $C_{y r}$ and $C_{z r}$ and its deposition profile are described by Eq. 18. The cylinder growth and the scalars $C_{y l}$ and $C_{z l}$ of the left region can be found in Eq. 19 .

In Eq.19), it can be noted that there is a limit $\theta_{2}$ for material deposition, because it is assumed that the contact between filaments is an obstacle to the material flow beneath the intersection point, therefore material can not be deposited below this point, as shown in Fig 11.a). This angle is calculated from Eq.20 and the dimensions $\bar{z}$ and $\bar{y}$ used to calculate $\theta_{2}$ are depicted in Fig 11(b). Moreover, in order to force continuity between the geometry of the left and right regions, it is assumed that $C_{z l}=C_{z r}$. By assuming this, the left and right regions always have the same height. This comes from the fact that no material discontinuity was observed between the left and right regions of the filament in experiments.

$$
\begin{aligned}
& \left\{\begin{array}{c}
x_{c}=x, \\
y_{c}=y_{0}+\left(R_{b}+C_{y r} \sin \left(\frac{\pi\left(\theta+\theta_{1}\right)}{2 \theta_{1}+\pi}\right)\right) \cos \theta, \\
z_{c}=z_{0}+\left(R_{b}+C_{z r} \sin \left(\frac{\pi\left(\theta+\theta_{1}\right)}{2 \theta_{1}+\pi}\right)\right) \sin \theta,
\end{array}\right. \\
& \text { for } \theta \in\left[-\theta_{1}, \pi / 2\left[, \quad x \in\left[x_{0}-L_{c} / 2, x_{0}+L_{c} / 2[\text {. }\right.\right.\right.
\end{aligned}
$$




$$
\left\{\begin{array}{c}
x_{c}=x \\
y_{c}=y_{0}+\left(R_{b}+C_{y l} \sin \left(\frac{\pi\left(\theta+\theta_{1}\right)}{2 \theta_{1}+\pi}\right)\right) \cos \theta \\
z_{c}=z_{0}+\left(R_{b}+C_{z l} \sin \left(\frac{\pi\left(\theta+\theta_{1}\right)}{2 \theta_{1}+\pi}\right)\right) \sin \theta
\end{array}\right.
$$

for $\theta \in\left[\pi / 2, \theta_{2}\left[, \quad x \in\left[x_{0}-L_{c} / 2, x_{0}+L_{c} / 2[\right.\right.\right.$.

$$
\theta_{2}=\pi-\tan ^{-1}\left(\frac{\bar{z}}{\bar{y}}\right) \text {. }
$$

Affected by old filament | Not affected by old filament

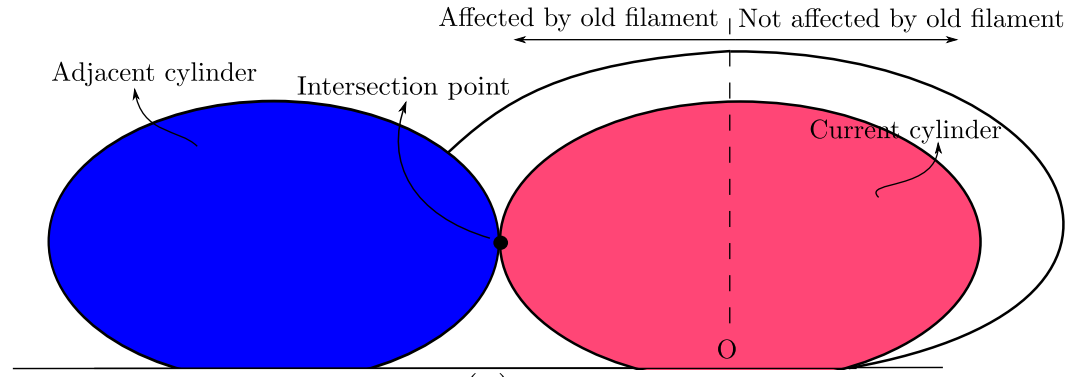

(a)

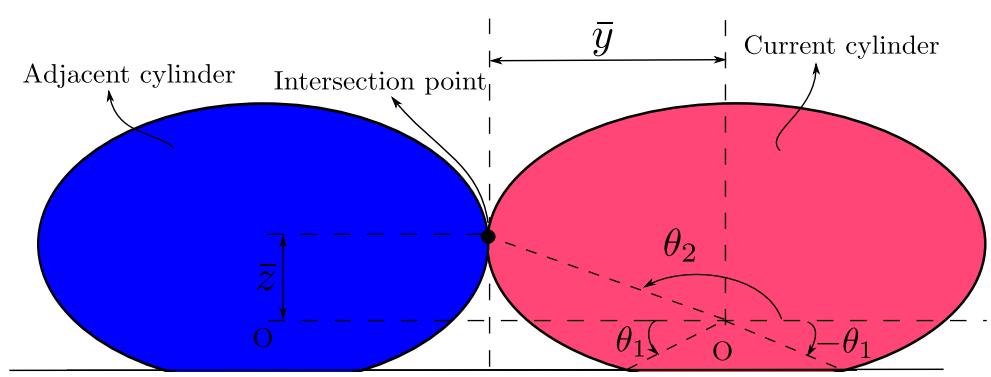

(b)

Figure 11: (a) Regions defined after the intersection of cylinders; (b) contact angle $\theta_{2}$ between the adjacent and current cylinder.

At the instant the adjacent and current cylinders intersect, the volume that still needs to be added to the model $\Omega^{\prime}$ to reach the target volume is given by Eq.21].

$$
\Omega^{\prime}=\Omega_{\text {target }}-\Omega_{\text {Total }}^{n}
$$

Considering that the filament exits the nozzle with a constant speed $V_{N}$ (see Fig 4) throughout the radius, it is reasonable to assume that the volume $\Omega^{\prime}$ will be distributed equally among the left and right regions of the filament: the left region will have its volume added by $\Omega^{\prime} / 2$ and the same shall occur to the right region. 
The following procedure is applied to find the scalars $C_{y r}, C_{z r}, C_{y l}$ and $C_{z l}$ :

1. Start with $C_{z l}=C_{z r}=0$;

2. At the right region of the cylinder, calculate $C_{y r}$ so that the volume of the entire structure reaches the value of $\Omega_{\text {target }}-\frac{\Omega^{\prime}}{2}$;

3. At the left region of the cylinder, increase $C_{y l}$ iteratively in order to reach the target volume of the entire structure $\Omega_{\text {target }}$. If the scalar $C_{y l}$ is increased in two consecutive iterations, but the volume of the entire structure has not increased, it means that for the current height there is no solution, since the adjacent cylinder may not allow increase in the $y$ direction;

4. If no solution is found at step 3, increase value of $C_{z l}$ and go to step 2 .

\subsection{Growth profile when the current cylinder touches the print nozzle}

It can be observed in the procedure described to calculate the scalars $C_{y r}, C_{z r}, C_{y l}$ and $C_{z l}$ that the scalar $C_{z l}$ is incremented until a solution is found. As observed in Eq. 19, when $C_{z l}$ is incremented, the height of the current cylinder increases. For large quantities of deposited material per filament, when applying the iterative procedure to calculate $C_{y r}, C_{z r}, C_{y l}$ and $C_{z l}$, there is the possibility of the current cylinder to touch the print nozzle. In this scenario, a different deposition modelling is employed.

Experiments were performed to evaluate the material distribution in cases with large quantities of deposited material per filaments. It was observed that when large quantities of material are deposited, almost no voids were observed between neighboring filaments. This indicates that material is forced to be deposited below the intersection point between neighboring filaments. The intersection point can be seen in Fig.10. Moreover, it was observed that there was material above and around the print nozzle.

\subsubsection{Material deposition at the void region}

At the moment the current cylinder touches the print nozzle, the total volume of the structure is defined by $\Omega_{\text {nozzle }}$. Therefore, the remaining volume that still needs to be deposited is given by $\Omega_{\mathrm{r}}=\Omega_{\text {target }}-\Omega_{\text {nozzle }}$.

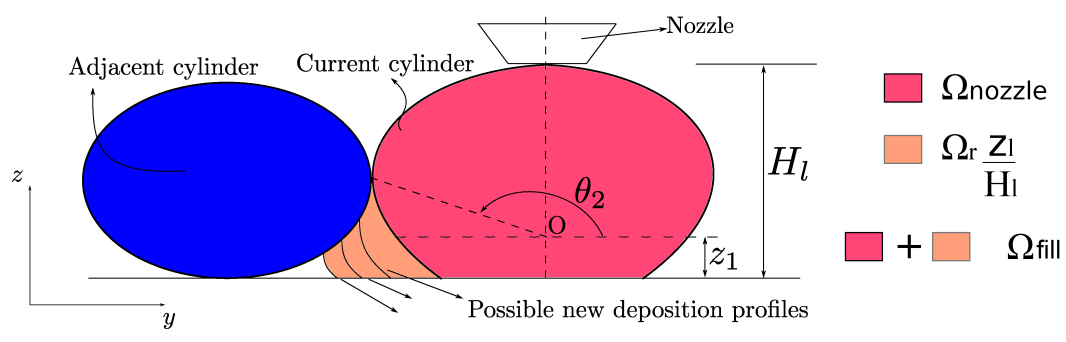

Figure 12: Deposition profile for the void region between neighboring filaments. 
In order to incorporate the phenomenon of low void volume fraction for high quantities of material being deposited, a void region between neighboring filaments is defined, as in Fig 12. A target volume for the void region is defined by $\Omega_{\text {fill }}$ in Eq. 222. $H_{l}$ is the distance between the nozzle and the layer below, and $z_{1}$ is the distance between the centre of the cylinder and the layer below, in $z$ direction, as observed in Fig 12 . The target volume at the void region is a proportion of the remaining volume $\Omega_{\mathrm{r}}$.

$$
\Omega_{\text {fill }}=\Omega_{\text {nozzle }}+\Omega_{\mathrm{r}} \frac{z_{1}}{H_{l}}
$$

The deposition profile for the void region can be seen in Fig 12 and can be calculated by Eq.23.

$$
\begin{gathered}
x_{c}=x, \\
\left\{\begin{array}{c}
y_{c}=y_{0}+\Delta R_{v} \frac{y_{r}}{z_{r}} \cos \theta, \\
z_{c}=z_{0}+\Delta R_{v} \sin \theta,
\end{array}\right.
\end{gathered}
$$

In Eq. (23), $\Delta R_{v}$ is the parameter that increases or decreases the shape of the cylinder at the void region. Then, at the simulation step $n$, the value $\Delta R_{v}$ is obtained by solving Eq.24) numerically, using the bisection method. In this equation, $\Omega_{\text {Total }}^{n}$ is the total volume of the printed part, which is function of $\Delta R_{v}$.

$$
\Omega_{\text {fill }}-\Omega_{\text {Total }}^{n}\left(\Delta R_{v}\right)=0 .
$$

\subsubsection{Material deposition above the print nozzle}

When a large amount of material is deposited, it is observed that material is deposited above the print nozzle. In VOLCO-X, when the current cylinder touches the print nozzle, a different material deposition is applied, as described in the following paragraphs.

First, the actual geometry of the nozzle with diameter $D_{N}(\mathrm{Fig} 13(\mathrm{a}))$ is simplified as in Fig 13 (b). In VOLCO-X, material can not be deposited within the region $\Theta_{n}$, which is the position occupied by the print nozzle.

Moreover, when the deposited material surpassed the nozzle height a flat surface was observed in printed specimens during experiments. Therefore, it was proposed the material deposition above the nozzle behaved as follows:

1. Start at position $\bar{y}_{n}=y_{0}^{i}-\frac{D_{N}}{2}\left(y_{0}^{i}\right.$ is the centre of the current filament $\left.i\right)$ and $\bar{z}_{n}=l_{t}\left(\right.$ layer $\left._{i}-1\right)$ 


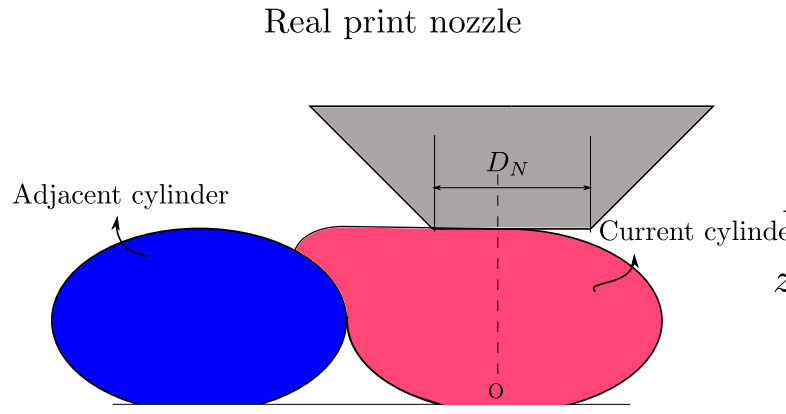

(a)

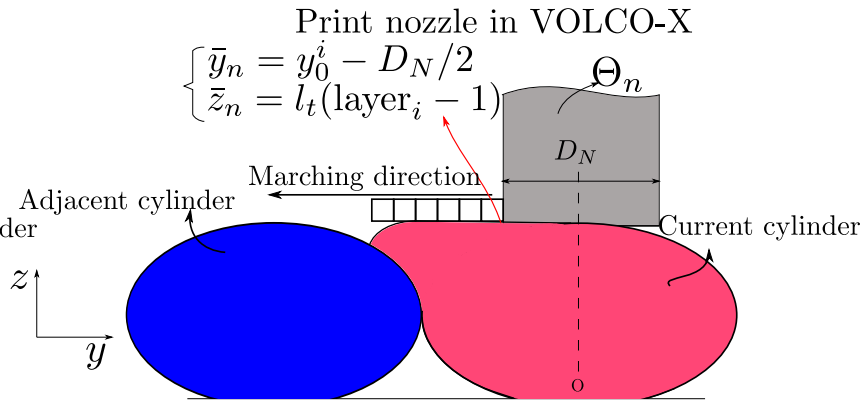

(b)

Figure 13: Material distribution modelling above the nozzle: (a) real geometry of the nozzle; (b) geometry of the nozzle in VOLCO-X and marching direction to fill the voids above the nozzle.

(position of the nozzle at layer ${ }_{i}$ ). The term $l_{t}$ is the layer thickness set in the.$g c o d e$. The coordinates $\bar{y}_{n}$ and $\bar{z}_{n}$ can be seen in Fig $\left.13(\mathrm{~b})\right)$;

2. Find the voxels which coordinates lay within the range of $y \in\left[y_{0}^{i-1}-\frac{D_{N}}{2}, \bar{y}_{n}\right]$ with $z=\bar{z}_{n}$. The term $y_{0}^{i-1}$ is the centre of the neighboring filament;

3. March iteratively in the opposite direction of vector $y$ (as in Fig.13) filling the voids in each iteration. If $\Omega_{\text {target }} \leq \Omega_{\text {Total }}^{n}$ for any iteration, the deposition process is complete. Otherwise, go to Step 4;

4. Select a new starting position $\bar{z}_{n} \equiv \bar{z}_{n}+V_{s}$, where $V_{s}$ is the voxel size, and go to Step 2 .

\section{VOLCO-X computational implementation}

VOLCO-X was implemented in Python ${ }^{\circledR}$ and accounts for the influence of the printing speed and distance between deposited filaments by implementing the modelling approaches explained in Sections 3 and 4 respectively. The simulation flowchart can be seen in Fig 14.

\subsection{Memory and simulation time}

The memory usage and the computation time required to run VOLCO-X simulations as function of the voxel size can be seen in Fig, 15. In this computational cost analysis, the simulations were carried out with 88 printed filaments which correspond to 88 split cylinders and a total deposited material volume of $805 \mathrm{~mm}^{3}$. It is observed that the simulation time increases when the voxel size decreases. Cleary, the relationship between voxel size and the time required to run a simulation is not linear: the simulation time was more than doubled when the voxel size was decreased in half. The same is observed for the required memory.

It can be seen that there is a memory peak at the beginning of the simulations because of the voxel matrix creation, initially filled with zero elements. Also, during the simulation, the coordinates of the outer 


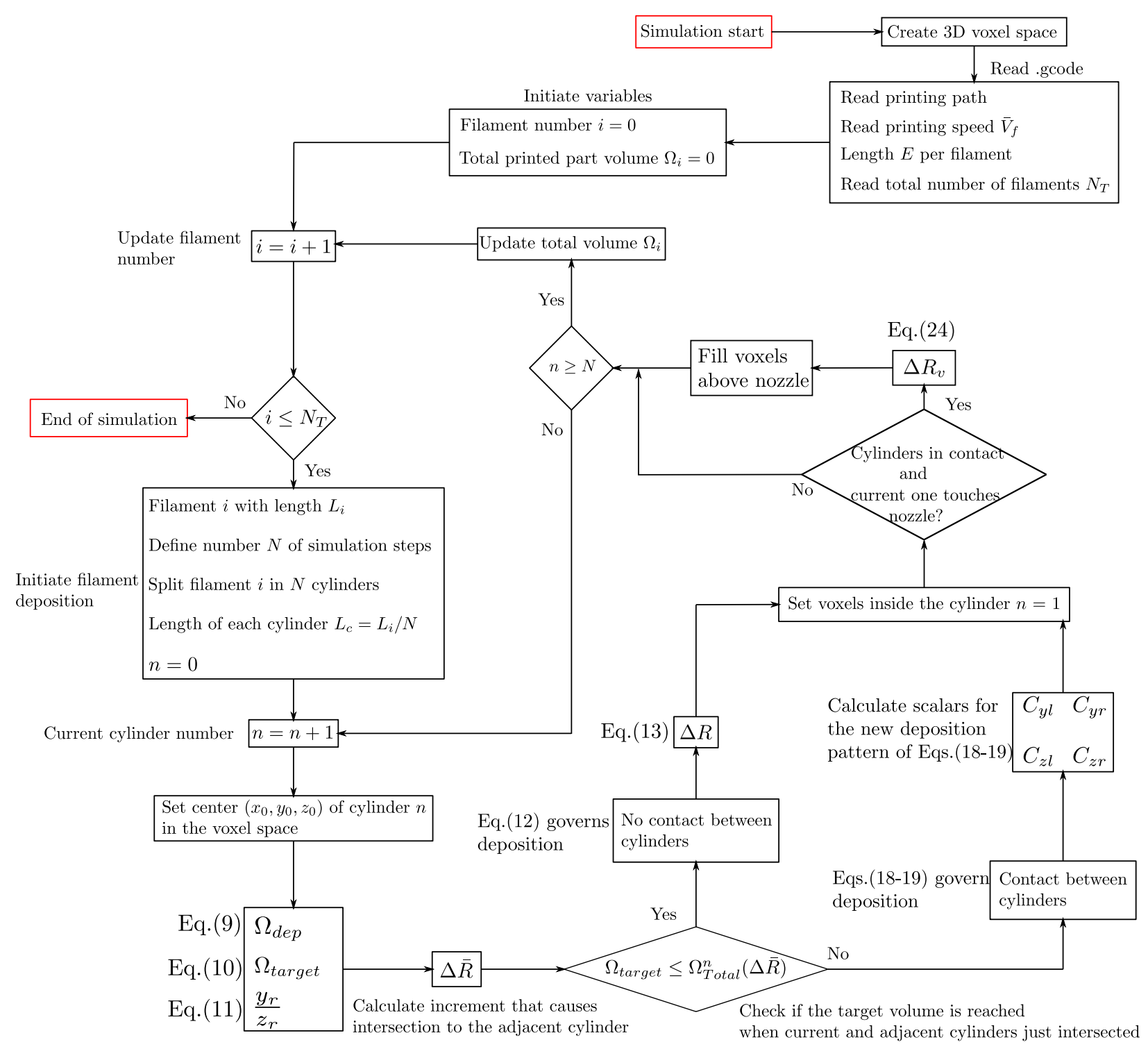

Figure 14: Flowchart simulation of VOLCO-X.

surface of the filaments are stored in arrays for contact computation. This is responsible for the increasing memory usage during the simulation.

\section{Methodology for simulations and experiments}

Simulations were carried out following the printing parameters described in Tab 1 . The 3D printer settings can also be found in this table. Three sets of simulations were performed using a voxel size of $5 \mu m m$.

In the first set of simulations, the influence of the printing speed on material distribution was investigated. 


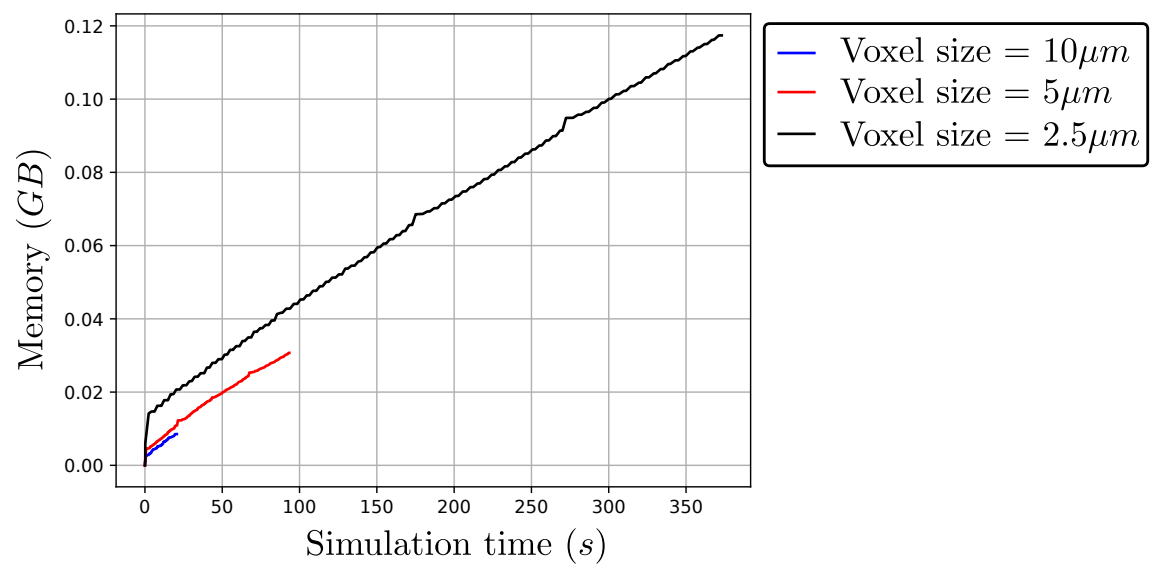

Figure 15: Simulation and memory usage as function of the voxel size.

For this, isolated filaments were simulated for two different printing speeds, $\bar{V}_{f}=16 \mathrm{~mm} / \mathrm{s}$ and $\bar{V}_{f}=$ $96 \mathrm{~mm} / \mathrm{s}$, with length $L=115 \mathrm{~mm}$ and $E=3.806 \mathrm{~mm}$ (considering filaments with initial diameter $D_{0}=$ $1.75 \mathrm{~mm}$, this value of $E$ represents a nominal volume of material to be extruded around $9.15 \mathrm{~mm}^{3}$.) Also, two specimens were simulated for these speeds, considering the distance between filaments $d=0.325 \mathrm{~mm}$ and $E$ per filament $E=3.806 \mathrm{~mm}$. The printer settings and printing parameters can be seen in Tab.1.

In the second set, specimens were simulated using the two printing speeds indicated in Tab $1\left(\bar{V}_{f}=\right.$ $16 \mathrm{~mm} / \mathrm{s}$ and $\left.\bar{V}_{f}=96 \mathrm{~mm} / \mathrm{s}\right)$. Four distances $(d=0.325 \mathrm{~mm}, d=0.3375 \mathrm{~mm}, d=0.35 \mathrm{~mm}$ and $d=$ $0.3625 \mathrm{~mm}$ ) between centres of deposited filaments were also employed in this set of simulations in order to analyse its effect on material distribution. The value of $E$ was fixed at $3.806 \mathrm{~mm}$ for this set of simulations.

In the third set, specimens were simulated using the same printing speeds $\bar{V}_{f}=16 \mathrm{~mm} / \mathrm{s}$ and $\bar{V}_{f}=$ $96 \mathrm{~mm} / \mathrm{s}$. However, the $E$ value was varied based on multiples of a base value of $\bar{E}=3.806 \mathrm{~mm}: E=0.95 \bar{E}$, $E=1.0 \bar{E}, E=1.2 \bar{E}$ and $E=1.5 \bar{E}$. For these simulations the distance between deposited filaments was fixed as $d=0.325 \mathrm{~mm}$. The printer settings and additional printing parameters used in the simulations can be seen in Tab1 It should be noted that when $E$ was changed, extrusion speed of material exiting the nozzle also changed proportionally. This speed change is automatically implemented by the printer when it interprets the value of $E$ set in GCode in order to control the feed of material into the hot end.

The printing path for each layer is depicted in Fig 16 . It can be observed that unidirectional printed parts were simulated for these tests of VOLCO-X. This was because the contact between deposited filaments plays a major role on the material distribution for these 3D printed parts. In this manner, it was possible to better evaluate the performance of VOLCO-X with the new proposed model for contact between filaments.

It is possible to estimate the cross sectional dimensions of specimens, based on the data given in Tab1. 
Table 1: Printer settings and printing parameters used to print specimens and in VOLCO-X simulations.

Printer settings

Nozzle diameter $(m m)-D_{N} \quad 0.4$

Nozzle acceleration $\left(\mathrm{mm} / \mathrm{s}^{2}\right)-a_{f} \quad 1200$

Extruder acceleration $\left(\mathrm{mm} / \mathrm{s}^{2}\right)-a_{e} \quad 1200$

Bulk filament diameter $(\mathrm{mm})-D_{0} \quad 1.75$

$X, Y, Z, E$ motors speed change threshold $(\mathrm{mm} / \mathrm{s}) \quad[40,40,40,5]$

Printing parameters

Layer thickness $(\mathrm{mm}) \quad 0.3$

Printing speed $(\mathrm{mm} / \mathrm{s})-\bar{V}_{f} \quad[16,96]$

Distance between filaments $(\mathrm{mm})-d \quad[0.325,0.3375,0.35,0.3625]$

$\bar{E}$ per filament $(\mathrm{mm}) \quad 3.806$

$E / \bar{E}$ per filament $\quad[0.95,1.0,1.2,1.5]$

Number of layers 4

Number of filaments per layer $\quad 22$

Filament length $(\mathrm{mm})-L \quad 114.858$

Nozzle temperature $\left({ }^{\circ} \mathrm{C}\right) \quad 210$

Bed temperature $\left({ }^{\circ} \mathrm{C}\right) \quad 110$

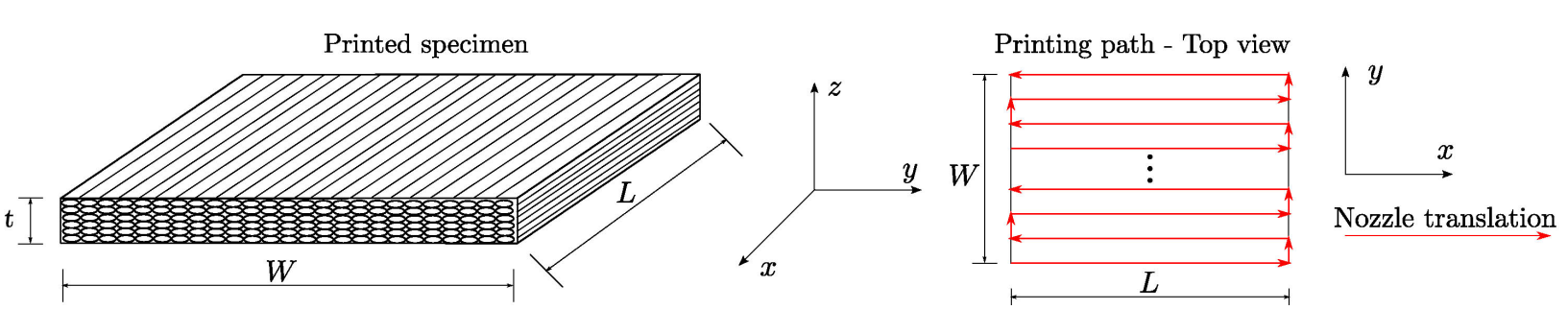

Figure 16: General specimens dimensions and printing path for each layer.

With several distances $d$ between printed filaments, 22 as number of filaments per layer, $0.3 \mathrm{~mm}$ as layer thickness and 4 as number of layers, nominal values for dimensions in Fig 16 can be estimated: $W$ in the range from 7.150 to $7.975 \mathrm{~mm}$ and $t=1.2 \mathrm{~mm}$. However, such dimensions vary according to actual deposited amounts of material, which vary with the aforementioned parameters $\bar{V}_{f}$ and $E$, as will be seen in the results section.

Experiments were performed to validate the simulation results. Specimens of ABS-MG94 (filament manufactured by Faz3D) were produced by FFF in an enclosed 3D printer (GTMax3D Core A2 @) following the same printing parameters in Tab 1 used for the VOLCO-X simulations.

For each combination of printing parameters in the second and third sets, three specimens were manufactured and had their dimensions measured at the central portion. In order to observe their microstructures at the lengthwise central portion of the specimens, they were cooled to $-90^{\circ} \mathrm{C}$ using a Vötsch machine and sectioned with a sharp blade. Micrographs were taken with a digital microscope. 


\section{Results and discussion}

In this section, numerical and experimental results are presented in view of the three sets of simulations detailed in Section 6. for single filaments, for multiple filaments with different printing distances $d$ and for multiple filaments with different extrusion length parameters $E$.

\subsection{Results of the first set of specimens (single filament)}

With the acceleration-dependent extrusion rate modelling approach explained in Section 3 simulations were carried out in VOLCO-X to investigate the shape of deposited filaments for different printing speeds. Filament profiles at different sections can be seen in Fig 17
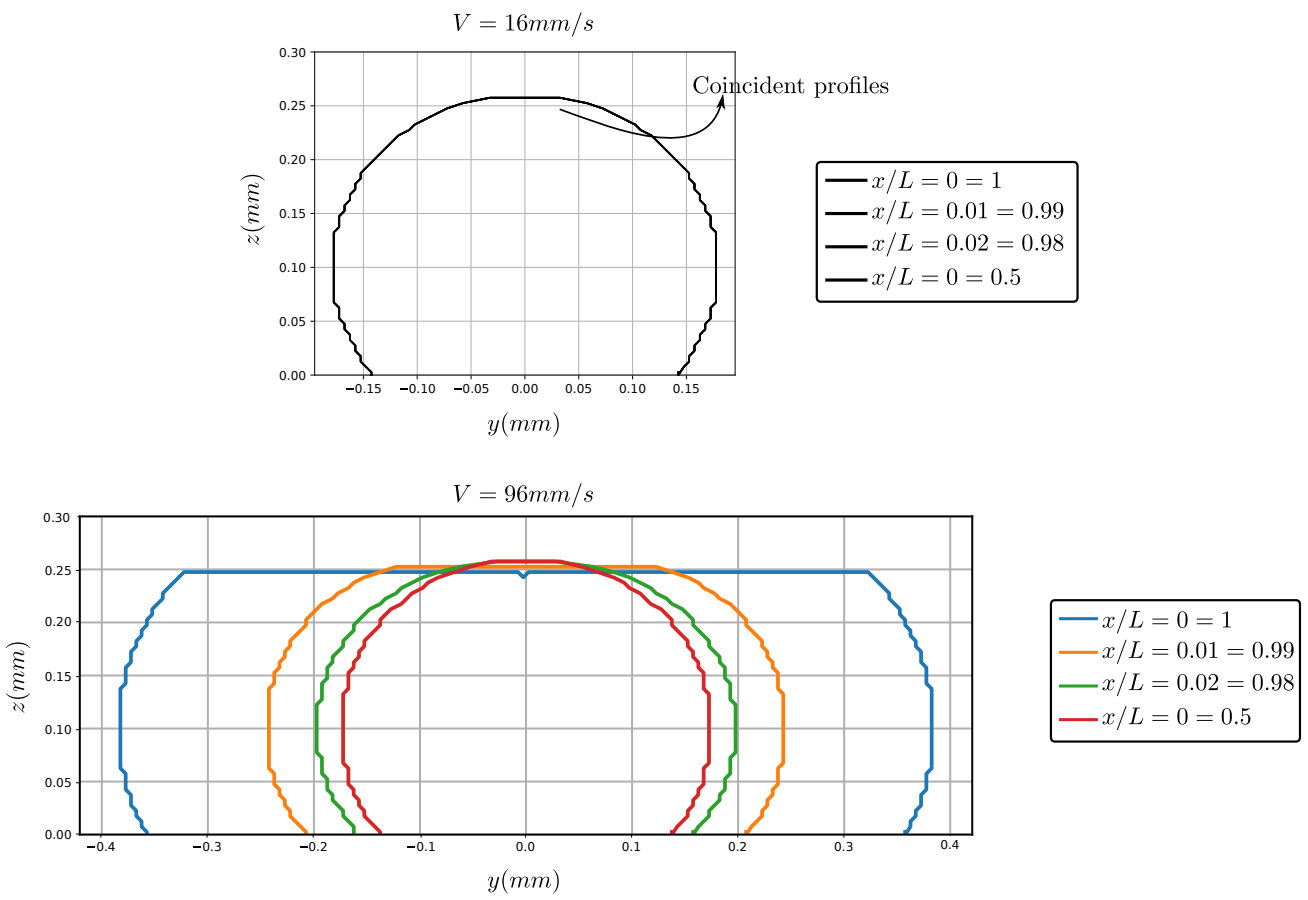

Figure 17: Numerical filament profiles at different cross sections of a single filament, printed with $\bar{V}_{f}=16 m m / s$ and $\bar{V}_{f}=$ $96 \mathrm{~mm} / \mathrm{s} . L$ is the length of the filament and $x$ is the coordinate of the analysed portion.

In this figure, it can be observed that when $\bar{V}_{f}=16 \mathrm{~mm} / \mathrm{s}$ the cross section of a single filament is constant throughout its length. However, when the printing speed was increased to $\bar{V}_{f}=96 \mathrm{~mm} / \mathrm{s}$, it is seen that a bigger filament width was observed at the starting $(x / L=0 ; 0.01 ; 0.02)$ and final $(x / L=1 ; 0.99 ; 0.98)$ filament cross sections. Note that $x=0$ signifies the initial portion of the filament and $x=L$ signifies the final portion of the filament.

The formation tendencies of filament profiles in Fig 17 were investigated using VOLCO-X, by observing the effect of the printing speed on the deposited volume of a single filament. Results for $\bar{V}_{f}=16 \mathrm{~mm} / \mathrm{s}$ and 
$\bar{V}_{f}=96 \mathrm{~mm} / \mathrm{s}$ can be observed in Fig $18(\mathrm{a}),(\mathrm{b}),(\mathrm{c})$ and $(\mathrm{d})$.

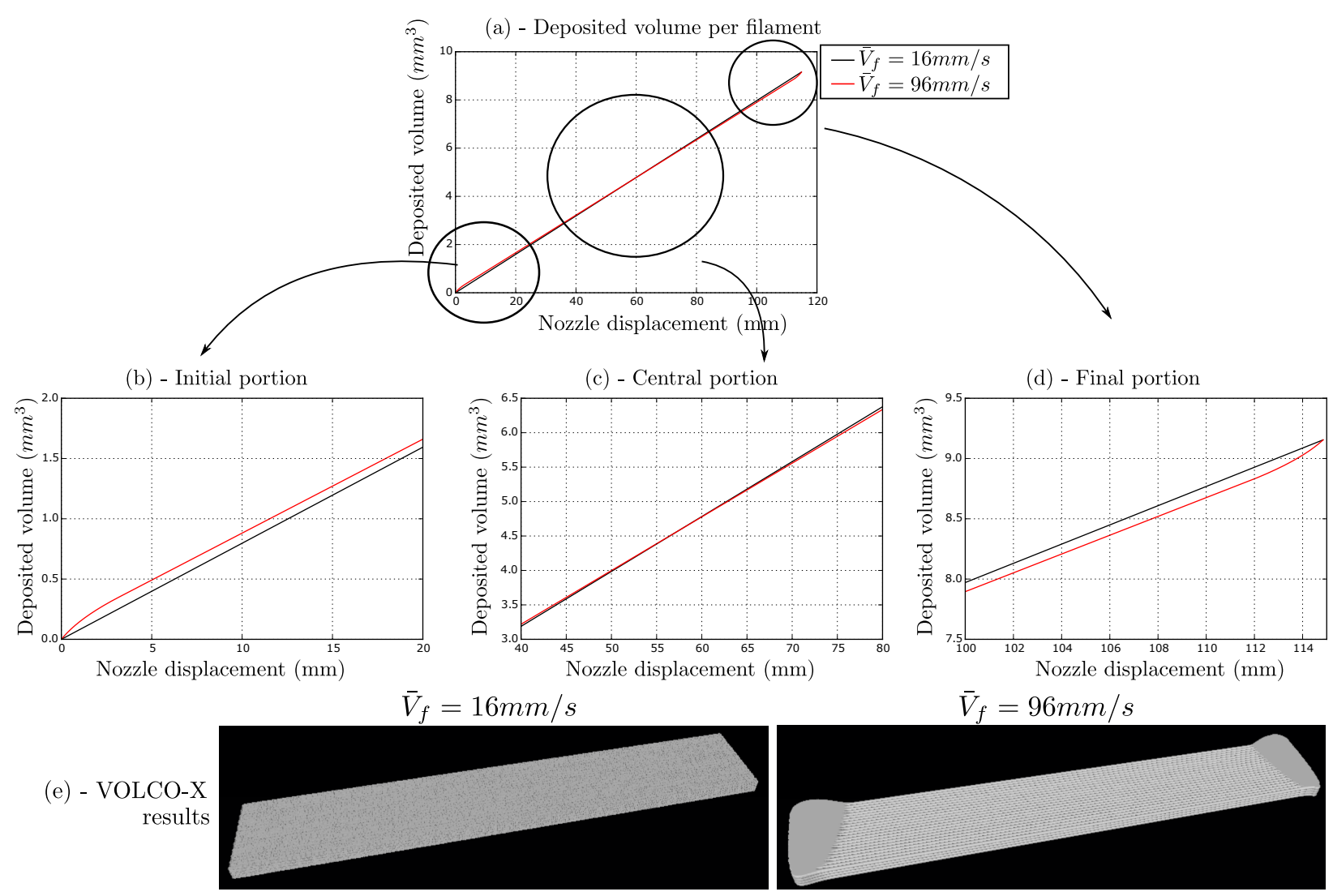

Figure 18: Deposited volume per filament simulated for two printing speeds: (a) entire filament; (b), (c) and (d) zoom at the initial, central and final portion of the filament, respectively; (e) full specimen simulated by VOLCO-X as a function of the printing speed.

When $\bar{V}_{f}=16 \mathrm{~mm} / \mathrm{s}$, the nozzle speed is constant, because $\bar{V}_{f}=16 \mathrm{~mm} / \mathrm{s}<V_{t}=40 \mathrm{~mm} / \mathrm{s}\left(V_{t}\right.$ being the speed change threshold of the nozzle). The extrusion speed (calculated by Eq.(5)) is also constant. Since these two speeds are constant, the volume deposited throughout the filament is also constant. This can be observed in Figs 18 (b), (c) and (d) as $d \Omega_{d} / d S$ is constant throughout all portions of the filament, $\Omega_{d}$ being the deposited volume and $S$ the displacement of the nozzle.

When $\bar{V}_{f}=96 \mathrm{~mm} / \mathrm{s}$, the nozzle speed is not constant, because $\bar{V}_{f}=96 \mathrm{~mm} / \mathrm{s}>V_{t}=40 \mathrm{~mm} / \mathrm{s}$. This means that the nozzle accelerates and decelerates in the initial and final portions of the filament, respectively, as seen in Fig.19. However, the extrusion speed $\left(V_{e}\right)$ is constant in time when calculated by Eq. (5) (as in Fig $5(\mathrm{~b} 1))$.

Some analytical expressions from Sec 3 are here revisited in order to further assess the numerical results 


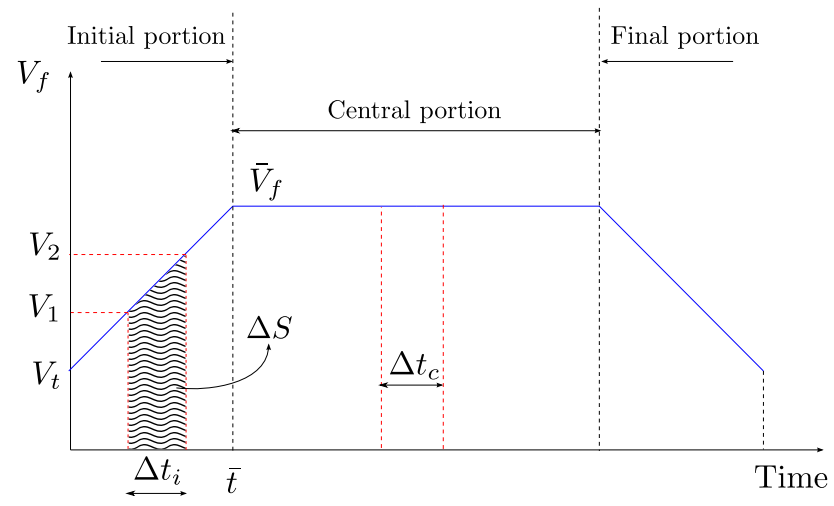

Figure 19: Speed profile for the nozzle when $\bar{V}_{f}=96 \mathrm{~mm} / \mathrm{s}$.

in Fig 17 and 18 Deposited material volume can be easily calculated by solving Eq. (9) as follows:

$$
\Omega_{d}=\bar{V}_{e} \int \pi \frac{D_{0}^{2}}{4} d t=\bar{V}_{e} \pi \frac{D_{0}^{2}}{4} t .
$$

At the central portion of the filament, the printing speed is constant at a value of $\bar{V}_{f}=96 \mathrm{~mm} / \mathrm{s}$. If the nozzle travelled a distance of $\Delta S$ during a period of time of $\Delta t_{c}$, the amount of deposited volume $\left(\Delta \Omega_{d}^{c}\right)$ is calculated by Eq.(26). The deposited volume per distance travelled by the nozzle at the central portion is given by Eq. 27$]$.

$$
\begin{gathered}
\Delta \Omega_{d}^{c}=\bar{V}_{e} \pi \frac{D_{0}^{2}}{4} \Delta t_{c}=\bar{V}_{e} \pi \frac{D_{0}^{2}}{4} \frac{\Delta S}{\bar{V}_{f}}, \\
\frac{\Delta \Omega_{d}^{c}}{\Delta S}=\frac{\bar{V}_{e} \pi D_{0}^{2}}{4 \bar{V}_{f}} .
\end{gathered}
$$

At the initial portion of the filament, the nozzle travels the same distance $\Delta S$ during a period of time $\Delta t_{i}$. The relationship between these variables is given by Eq. 287 , which can be obtained by calculating the area under the curve depicted in Fig 19

$$
\Delta S=\frac{\left(V_{1}+V_{2}\right) \Delta t_{i}}{2}
$$

The volume of material deposited at the initial portion of the filament $\left(\Omega_{d}^{i}\right)$ when the nozzle travelled $\Delta S$ is given by Eq. 29$]$. The ratio between these two variables is given by Eq. 30$]$.

$$
\Delta \Omega_{d}^{i}=\bar{V}_{e} \pi \frac{D_{0}^{2}}{4} \Delta t_{i}=\bar{V}_{e} \pi \frac{D_{0}^{2}}{4} \frac{2 \Delta S}{V_{1}+V_{2}},
$$




$$
\frac{\Delta \Omega_{d}^{i}}{\Delta S}=\frac{\bar{V}_{e} \pi D_{0}^{2}}{2\left(V_{1}+V_{2}\right)}
$$

Finally, it is possible to compare the volume of material deposited at the initial and central portions of the filament by Eq. 31].

$$
\frac{\left(\Delta \Omega_{d}^{i} / \Delta S\right)}{\left(\Delta \Omega_{d}^{c} / \Delta S\right)}=\frac{2 \bar{V}_{f}}{V_{1}+V 2}
$$

It can be seen that $V_{1}+V_{2}<2 \bar{V}_{f}$ for any $\left(V_{1}, V_{2}\right)$ combination in the region where $t<\bar{t}$. This results in the relationship of Eq. 32, which means that more volume of material is deposited at the initial portion of the filament than the central one. The same can be demonstrated for the final portion of the filament. Therefore, it can be concluded that for high printing speeds (when $\bar{V}_{f}>V_{t}$ ), less material is deposited at the central portion of the filaments, leading to higher void volume fractions at the central portion.

$$
\frac{\Delta \Omega_{d}^{i}}{\Delta S}>\frac{\Delta \Omega_{d}^{c}}{\Delta S}
$$

The result above explains why the slopes $d \Omega_{d} / d S$, when $\bar{V}_{f}=96 \mathrm{~mm} / \mathrm{s}$, at the initial and final portions of the filament (Figs 18(b) and (d)) are different from the one observed at the central portion of the filament (Fig $18(\mathrm{c}))$.

In Fig 18 , taking into account a given nozzle displacement $\Delta S$ at the initial and final portions of the filament, a higher variation in deposited volume $\Delta \Omega_{d}$ was found for the highest printing speed $\left(\bar{V}_{f}=\right.$ $96 \mathrm{~mm} / \mathrm{s})$, i.e., $\left(d \Omega_{d} / d S\right)_{\bar{V}_{f}=96}>\left(d \Omega_{d} / d S\right)_{\bar{V}_{f}=16}$. This means that the volume deposited at the initial and final portions of the filament printed with $\bar{V}_{f}=96 \mathrm{~mm} / \mathrm{s}$ was greater than that printed with $\bar{V}_{f}=16 \mathrm{~mm} / \mathrm{s}$.

Moreover, the same behaviour can be seen in the VOLCO-X results for the full multi filament specimens depicted in Fig 18 (e), where there was more material at the initial and final portions of the specimen when $\bar{V}_{f}=96 \mathrm{~mm} / \mathrm{s}$, but the material distribution was constant when $\bar{V}_{f}=16 \mathrm{~mm} / \mathrm{s}$. The same tendency of material distribution was observed in full specimens experimentally manufactured as depicted in Fig 20 there is more material at the initial and final portions of the specimen printed with $\bar{V}_{f}=96 \mathrm{~mm} / \mathrm{s}$.

\subsection{Results of the second set of specimens (multiple filaments and varying d)}

The numerical and experimental results for the second set of specimens are presented below in which there are 22 filaments per layer. In the simulations and experiments, the distance between deposited filaments and the printing speed were varied. 

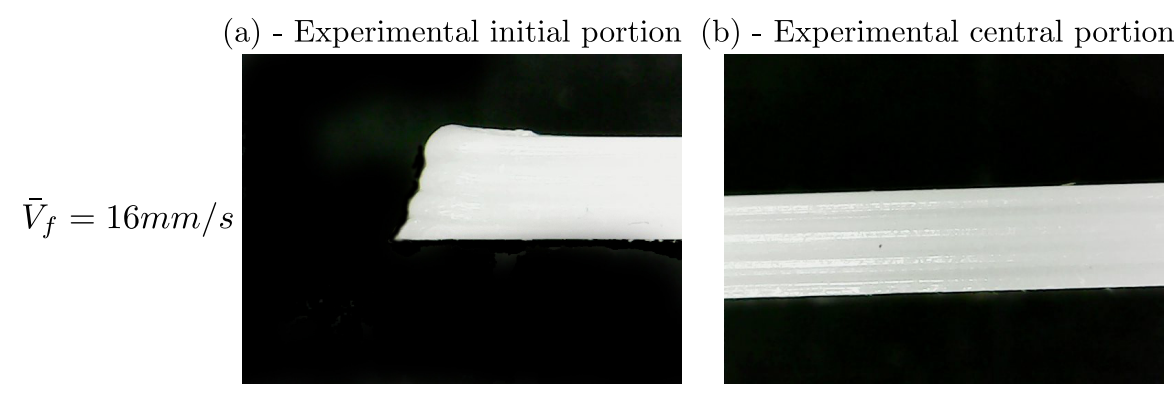

(c) - Experimental final portion
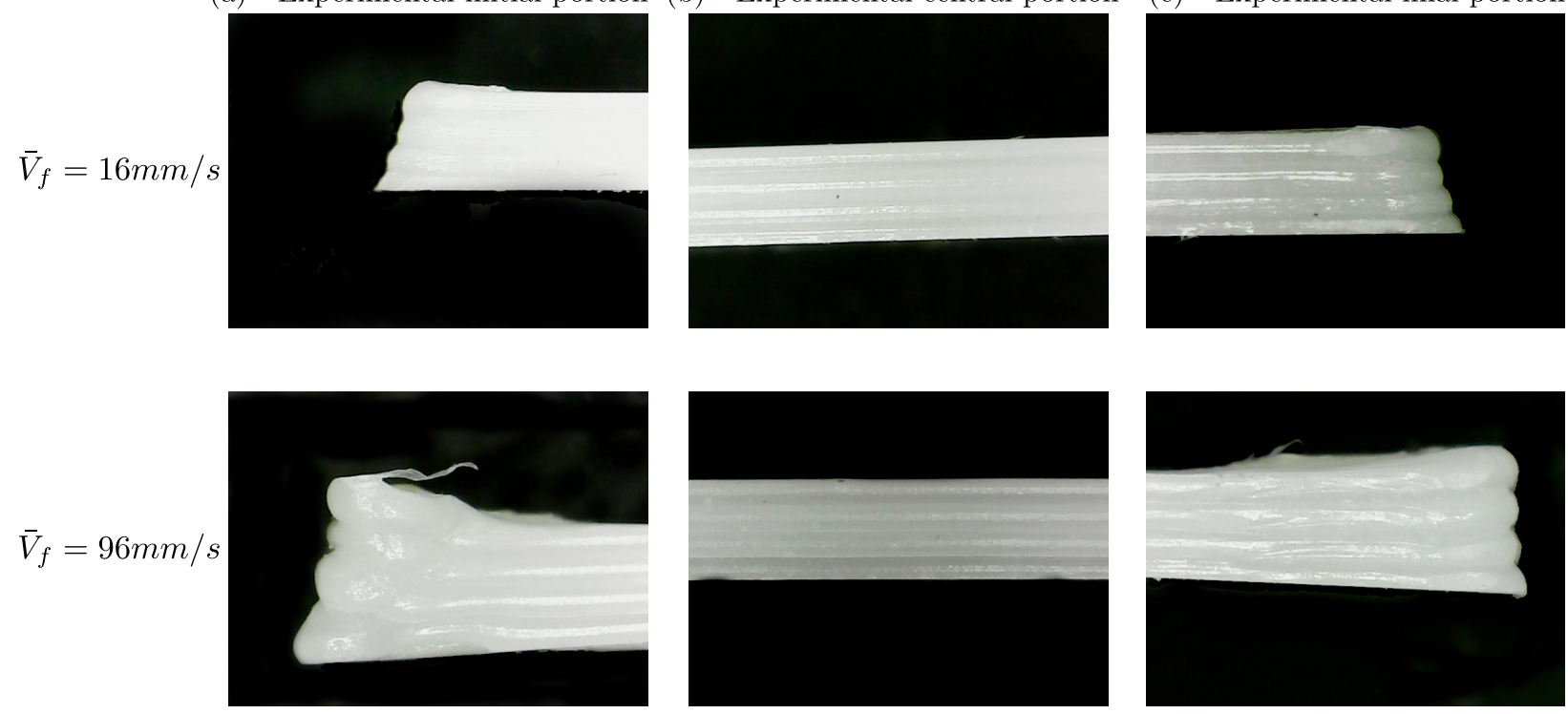

Figure 20: Material distribution along the thickness of specimens experimentally manufactured as a function of the printing speed at the initial, central and final portions.

\subsubsection{Numerical and experimental dimensions}

The numerical and experimental width and thickness (Tab2) at the center of the specimens is a function of the printing speed and distance between centres of deposited filaments. In the experiments, a monotonic increase of width with distance between deposited filaments was found and this tendency was also observed in the simulation results. VOLCO-X was able to predict the width and thickness with good agreement to experimental data: the largest differences between numerical and experimental results were $1.88 \%$ and $3.31 \%$ for the width and thickness, respectively.

Table 2: Dimensions of the specimens obtained numerically and experimentally. $d$ represents the distance between deposited filaments. $w$ and $t$ are the width and thickness of the printed part. $\Delta w$ and $\Delta t$ are the differences between experimental and numerical results for the width and thickness. $\Delta=(\mathrm{NUM}-\mathrm{EXP}) / \mathrm{NUM}$.

\begin{tabular}{cl|ll|llll} 
& & \multicolumn{2}{|l|}{ VOLCO-X } & & Experimental & & \\
& $d(\mathrm{~mm})$ & $w(\mathrm{~mm})$ & $t(\mathrm{~mm})$ & $w(\mathrm{~mm})$ & $\Delta w(\%)$ & $t(\mathrm{~mm})$ & $\Delta t(\%)$ \\
\cline { 2 - 7 } $\bar{V}_{f}=16 \mathrm{~mm} / \mathrm{s}$ & 0.325 & 7.19 & 1.055 & $7.25 \pm 0.04$ & -0.83 & $1.09 \pm 0.01$ & -3.31 \\
& 0.3375 & 7.46 & 1.025 & $7.49 \pm 0.04$ & -0.40 & $1.06 \pm 0.01$ & -3.41 \\
& 0.35 & 7.71 & 1.055 & $7.74 \pm 0.09$ & -0.38 & $1.07 \pm 0.01$ & -1.42 \\
& 0.3625 & 7.98 & 1.025 & $8.13 \pm 0.02$ & -1.88 & $1.03 \pm 0.01$ & -0.49 \\
\cline { 2 - 7 } $\bar{V}_{f}=96 \mathrm{~mm} / \mathrm{s}$ & 0.325 & 7.18 & 1.025 & $7.16 \pm 0.03$ & 0.27 & $1.06 \pm 0.01$ & -3.41 \\
& 0.3375 & 7.44 & 1.035 & $7.38 \pm 0.08$ & 0.80 & $1.06 \pm 0.01$ & -2.41 \\
& 0.35 & 7.7 & 1.025 & $7.75 \pm 0.04$ & -0.65 & $1.01 \pm 0.02$ & 1.46 \\
& 0.3625 & 7.96 & 1.025 & $8.04 \pm 0.02$ & -1.01 & $1.02 \pm 0.01$ & 0.49 \\
\hline
\end{tabular}




\subsubsection{Micrographs}

Micrographs at the central cross section of the simulated and experimental specimens manufactured with $\bar{V}_{f}=16 \mathrm{~mm} / \mathrm{s}$ as a function of the distance between centres of deposited filaments $d$ can be seen in Fig.21, The simulations were able to capture the void shapes experimentally obtained: triangles, skewed triangles, diamonds and gap faults. Moreover, the transition from triangular to diamond void shape observed in the experimental micrographs was captured by VOLCO-X. When $d=0.325 \mathrm{~mm}$, a mix of triangle and skewed triangle voids are seen. With an increase in distance $(d=0.3375 \mathrm{~mm})$ a more predominant skewed triangle shape was observed. For $d=0.35 \mathrm{~mm}$, diamond-like void shapes were obtained.

In the predicted micrograph, when $d=0.3625 \mathrm{~mm}$, there was no contact between filaments in the same layer. The experiments revealed gaps between filaments and a poor printing quality. This shows that VOLCO-X can predict the onset of inconsistent printing.

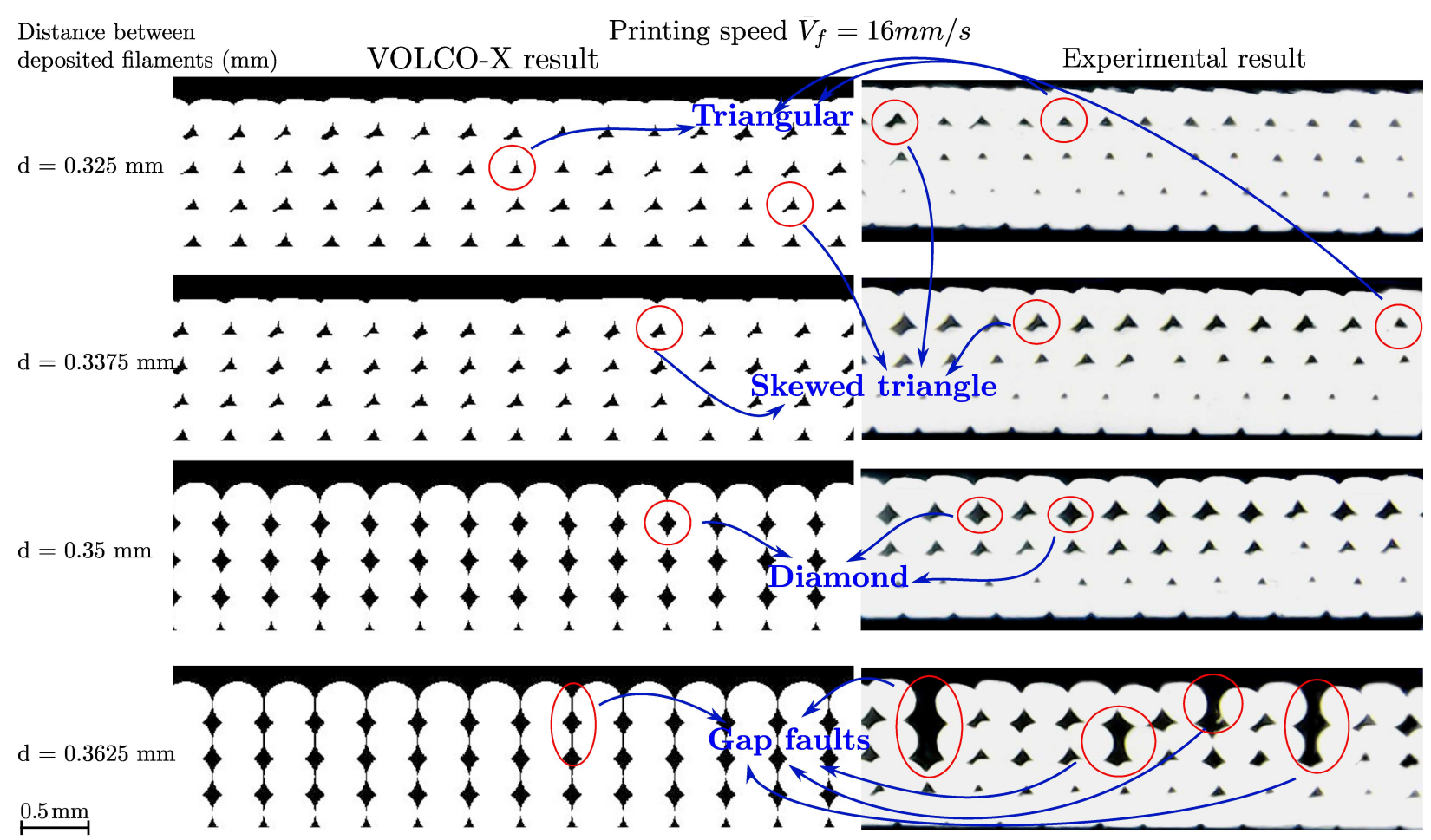

Figure 21: Printing speed $\bar{V}_{f}=16 \mathrm{~mm} / \mathrm{s}$ : experimental and numerical micrographs as a function of the distance between deposited filaments $d$. Micrographs at $x / L=0.5$.

Good agreement between simulations and experiments was also found for $\bar{V}_{f}=96 \mathrm{~mm} / \mathrm{s}$, as can be observed from the central cross section micrographs of the specimens shown in Fig 22, It can be seen that the void shapes (triangles, skewed triangles, diamond and gap faults) from VOLCO-X were in excellent agreement with the experimental ones. Moreover, the transition from triangular to diamond void shape (as 


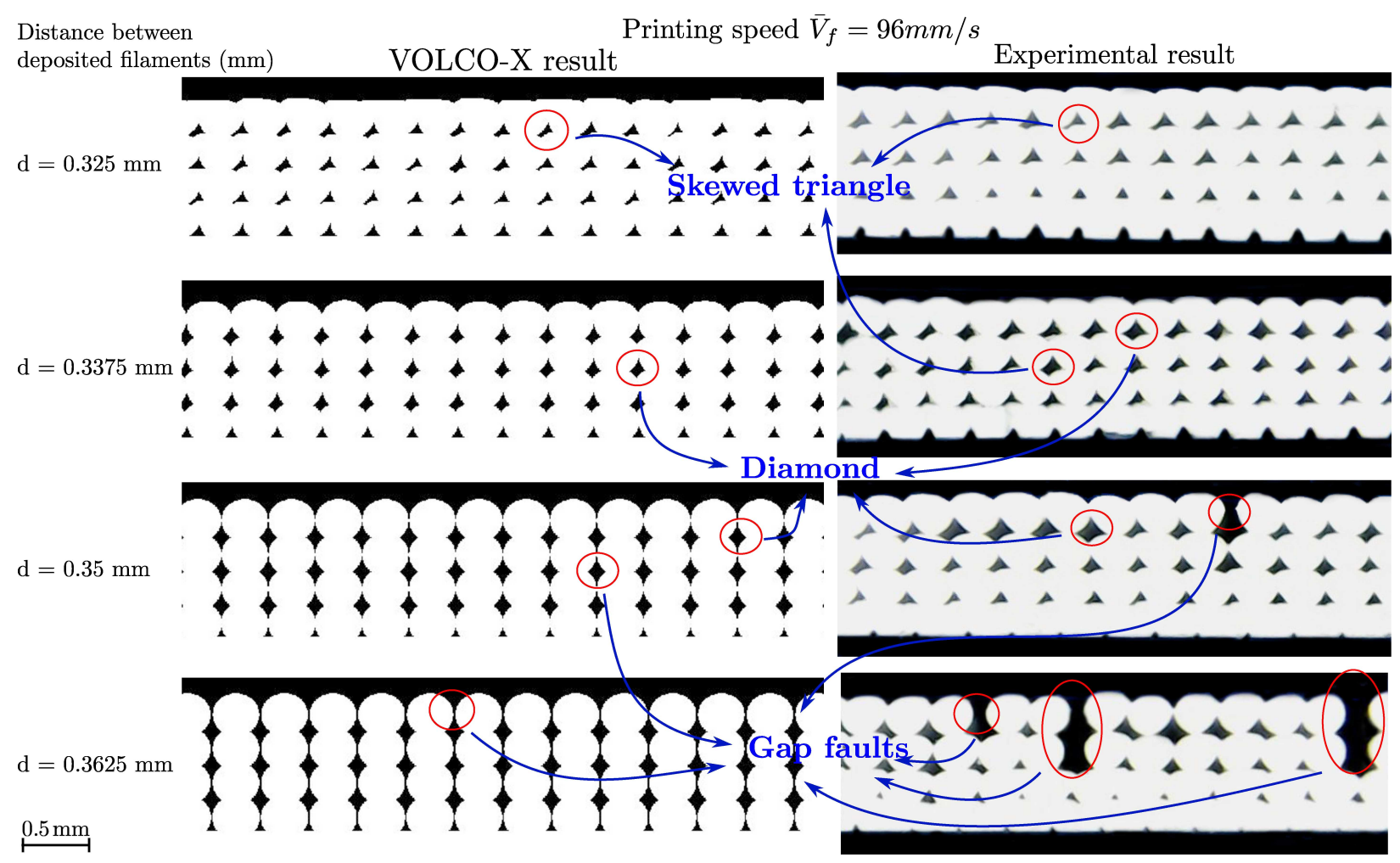

Figure 22: Printing speed $\bar{V}_{f}=96 \mathrm{~mm} / \mathrm{s}$ : experimental and numerical micrographs as a function of the distance between deposited filaments $d$. Micrographs at $x / L=0.5$.

filament spacing increased) was also in agreement with the experiments. Observing the experimental results for $\bar{V}_{f}=96 \mathrm{~mm} / \mathrm{s}$, it is possible to see that diamond void shapes were found for $d=0.3375 \mathrm{~mm}$ in contrast to Fig 21. where this void shape was found for $d=0.35 \mathrm{~mm}$ (when $\bar{V}_{f}=16 \mathrm{~mm} / \mathrm{s}$ ). These subtle differences were captured by the simulation results, showing that VOLCO-X is able to predict slight differences in material distribution within the printed part, which is useful to design functional printed parts with good accuracy.

\subsubsection{Void volume fraction}

A routine was implemented in MatLab to count the number of black (voids) and white (material) pixels in order to calculate the void volume fraction $\rho_{x}$. The simulation and experimental results can be seen in Fig.23. It can be seen in Fig 23(a) that the numerical $\rho_{x}$ presented a good correlation to the experimental data. VOLCO-X also accurately predicted the tendency of increase in void volume fraction when the distance between centre of filaments is increased.

In Fig $23(\mathrm{~b})$, for $\bar{V}_{f}=96 \mathrm{~mm} / \mathrm{s}$, the numerical void volume fraction presented a good correlation to the experimental data. Moreover, for this printing speed, more material was deposited at the initial and 
final portions of the specimens, as demonstrated in Section 7.1. Therefore, less material is expected to be deposited at the central portion of the specimen. In the experimental results, this was seen in the increase of void volume fraction when $\bar{V}_{f}=96 \mathrm{~mm} / \mathrm{s}\left(\rho_{16}<\rho_{96}\right)$. The same tendency was observed in the numerical results, showing that the simulation results are, again, in agreement with the experiments.

\section{Experimental data}

- VOLCO-X result

(b) $-\bar{V}_{f}=96 \mathrm{~mm} / \mathrm{s}$
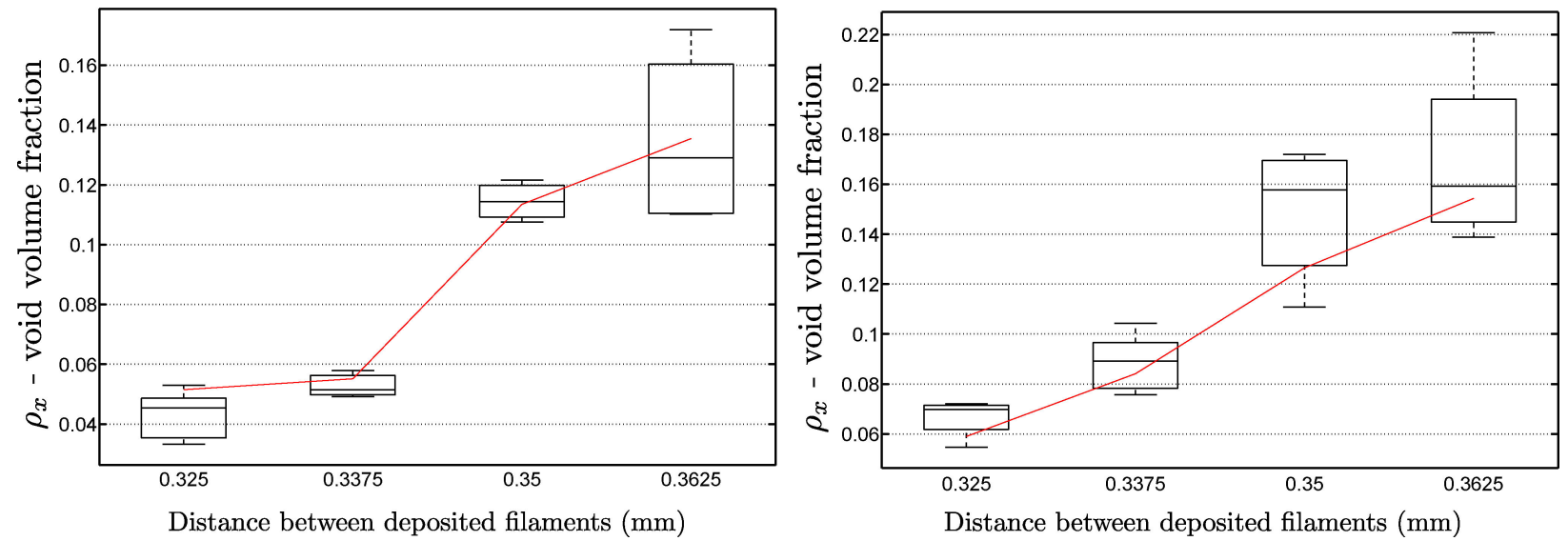

Figure 23: Experimental and numerical void volume fractions $\left(\rho_{x}\right)$ as function of $d$ for $\bar{V}_{f}=16 \mathrm{~mm} / \mathrm{s}$ and $\bar{V}_{f}=96 \mathrm{~mm} / \mathrm{s}$.

\subsection{Results of the third set of specimens (multiple filaments and varying E)}

The numerical and experimental results for the third set of specimens are presented below. In the simulations and experiments, the $E$ per deposited filament and the printing speed were varied.

\subsubsection{Numerical and experimental dimensions}

A comparison between numerical and experimental dimensions can be seen in Tab. 3 A monotonic increase in width and thickness with $E$ can be observed, which is expected, because more material is deposited when $E$ increases, causing an increase on the overall dimensions of the printed part. Again, there is good agreement between the VOLCO-X results and experimental data. For speed $\bar{V}_{f}=16 \mathrm{~mm} / \mathrm{s}$, the moduli of relative errors stayed below $1.5 \%$ for widths and below $5.5 \%$ for thicknesses. For speed $\bar{V}_{f}=96 \mathrm{~mm} / \mathrm{s}$, such errors remained at the most $2.55 \%$ for widths and below $4.5 \%$ for thicknesses, except for the result with $E / \bar{E}=1.5$. This discrepancy between VOLCO-X and experimental results is discussed in the following paragraphs. 
Table 3: Dimensions of the specimens obtained numerically and experimentally, with $\bar{E}=3.806 . w$ and $t$ are the width and thickness of the printed part. $\Delta w$ and $\Delta t$ are the differences between experimental and numerical results for the width and thickness. $\Delta=(\mathrm{NUM}-\mathrm{EXP}) / \mathrm{NUM}$.

\begin{tabular}{cl|ll|llll} 
& $E / \bar{E}$ & $w(\mathrm{~mm})$ & $t(\mathrm{~mm})$ & $w(\mathrm{~mm})$ & $\Delta w(\%)$ & $t(\mathrm{~mm})$ & $\Delta t(\%)$ \\
\cline { 2 - 7 } $\bar{V}_{f}=16 \mathrm{~mm} / \mathrm{s}$ & 0.95 & 7.18 & 1.025 & $7.23 \pm 0.04$ & -0.7 & $1.08 \pm 0.01$ & -5.36 \\
& 1.0 & 7.19 & 1.055 & $7.25 \pm 0.04$ & -0.83 & $1.09 \pm 0.01$ & -3.31 \\
& 1.2 & 7.26 & 1.215 & $7.32 \pm 0.02$ & -0.82 & $1.27 \pm 0.01$ & -4.52 \\
& 1.5 & 7.45 & 1.480 & $7.55 \pm 0.02$ & -1.34 & $1.48 \pm 0.01$ & 0.0 \\
\hline $\bar{V}_{f}=96 \mathrm{~mm} / \mathrm{s}$ & 0.95 & 7.17 & 1.025 & $7.11 \pm 0.02$ & 0.83 & $1.07 \pm 0.01$ & -4.39 \\
& 1.0 & 7.18 & 1.025 & $7.16 \pm 0.03$ & 0.27 & $1.06 \pm 0.01$ & -3.41 \\
& 1.2 & 7.24 & 1.205 & $7.21 \pm 0.02$ & 0.41 & $1.18 \pm 0.02$ & 2.07 \\
& 1.5 & 7.43 & 1.445 & $7.24 \pm 0.04$ & 2.55 & $1.23 \pm 0.1$ & 14.87 \\
\hline
\end{tabular}

\subsubsection{Micrographs}

It can be seen in Fig 24 that VOLCO-X was able to predict the shape of the voids with good agreement to the experimental micrographs for $\bar{V}_{f}=16 \mathrm{~mm} / \mathrm{s}$. With an increase in $E$, the voids ranged from skewed triangles to triangular void shapes. When $E / \bar{E}=1.5$, the amount of deposited material was enough to fill the voids between filaments and VOLCO-X was able to predict the scenario of almost no voids (fully dense structure) present in experimental micrographs.

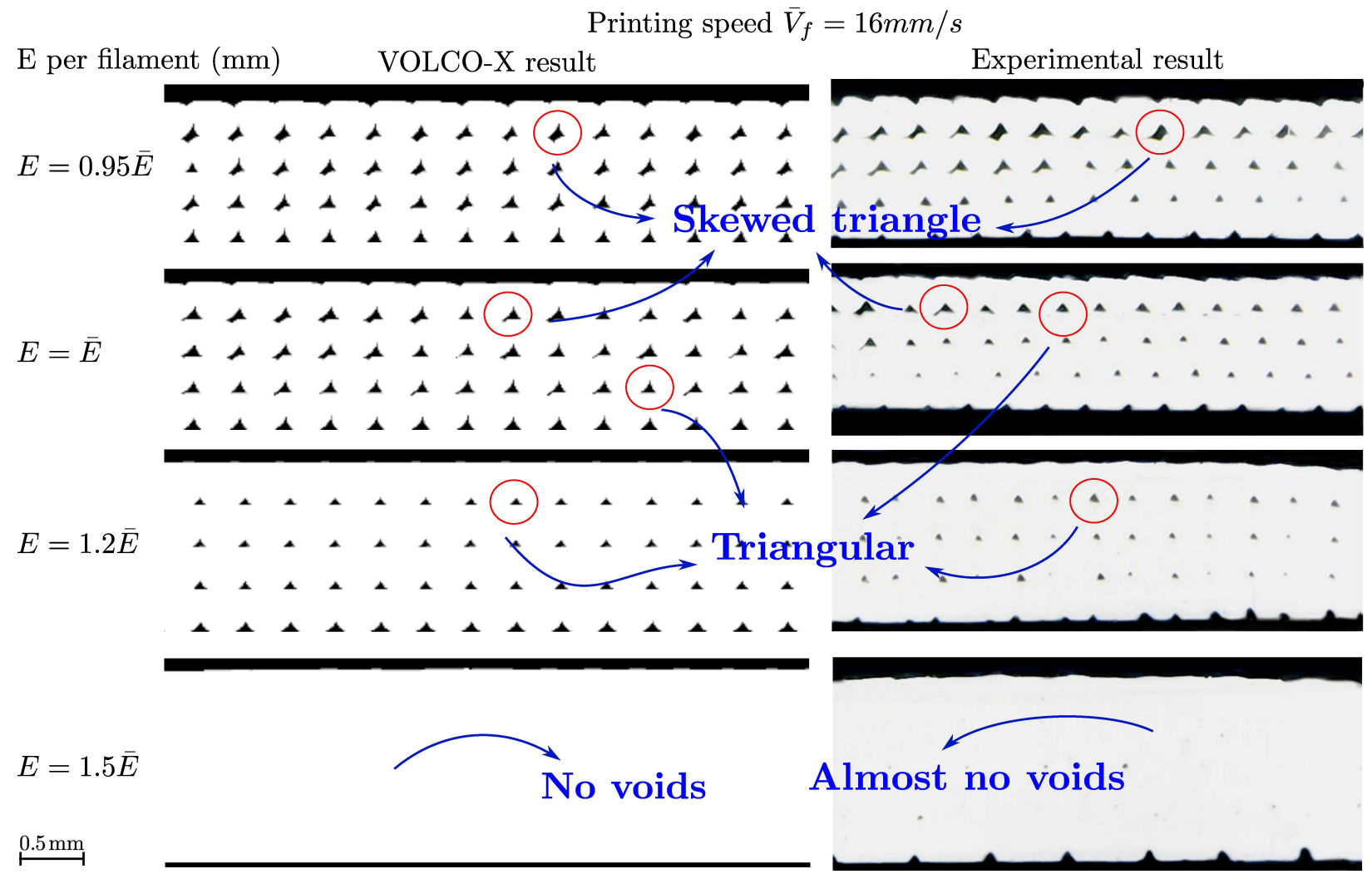

Figure 24: Printing speed $\bar{V}_{f}=16 \mathrm{~mm} / \mathrm{s}$ : experimental and numerical micrographs as a function of $E$. Micrographs at $x / L=0.5$. 


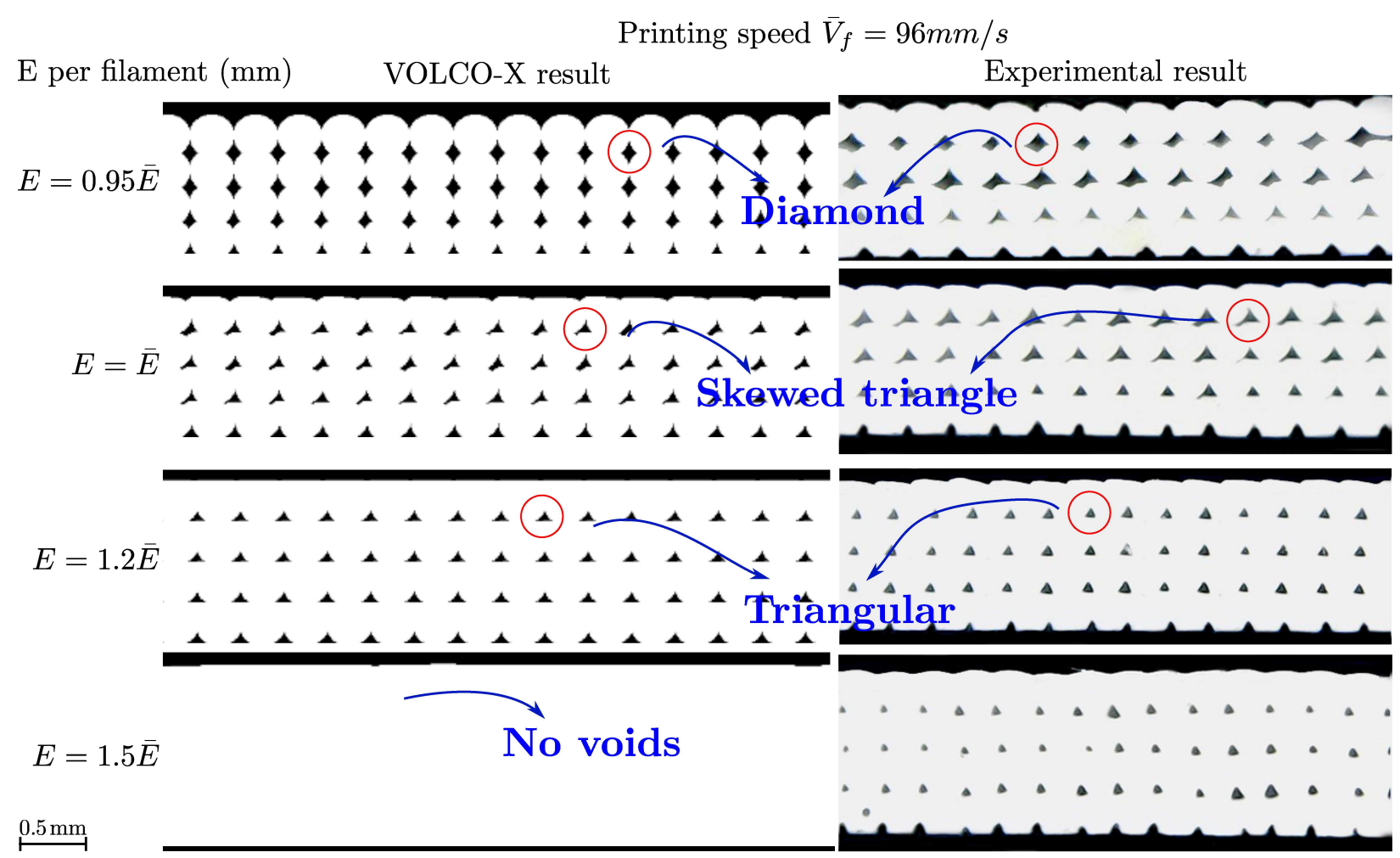

Figure 25: Printing speed $\bar{V}_{f}=96 \mathrm{~mm} / \mathrm{s}$ : experimental and numerical micrographs as a function of $E$. Micrographs at $x / L=0.5$.

The numerical void shapes for $\bar{V}_{f}=96 \mathrm{~mm} / \mathrm{s}$ depicted in Fig 25 presented good agreement to the experimental micrographs, except when $E / \bar{E}=1.5$. In this scenario, because of the high printing speed $\left(\bar{V}_{f}=96 \mathrm{~mm} / \mathrm{s}\right)$ and the large amount of extruded material $(E / \bar{E}=1.5)$, the extrusion speed $\left(V_{e}\right)$ was the highest among all performed experiments. With the increase of extrusion speed, the gears of the extruder motor had to turn quicker, causing slippage between gears, resulting in less material being deposited. This was confirmed by comparing the estimated design mass of the printed part $\left(M_{\text {design }}=1.304 \mathrm{~g}\right)$ and the actual mass of the printed part $\left(M_{\text {experiment }}=0.972 g\right)$ : the slippage of the extruder gears resulted in a printed part with $25.4 \%$ less mass.

In this manner, the differences in width and thickness observed for $\bar{V}_{f}=96 \mathrm{~mm} / \mathrm{s}$ and $E / \bar{E}=1.5$ in Tab 3 were caused by the reduction of material being deposited, reducing the actual width and thickness of the printed part. Moreover, because of the slippage of the extruder gears, the actual amount of deposited material was not enough to fill the voids between filaments as observed in the experimental micrographs in Fig 25, while no voids were observed in the numerical ones.

When $E / \bar{E}=0.95$, although the same amount of material was deposited for $\bar{V}_{f}=16 \mathrm{~mm} / \mathrm{s}$ and $\bar{V}_{f}=$ 
$96 \mathrm{~mm} / \mathrm{s}$, different void shapes were observed in the experimental micrographs: skewed triangles for the lowest and diamonds for the highest printing speed. This is because more material was deposited at the initial and final portions of the filaments when $\bar{V}_{f}=96 \mathrm{~mm} / \mathrm{s}$, resulting in less material at the central portion of the printed part, therefore diamond shapes were predominant at the lengthwise central portion.

It is worth mentioning that VOLCO-X was able to predict these subtle changes in void shapes as observed in Figs 24 and 25, demonstrating that the proposed numerical method is robust enough to capture slight changes in material distribution within printed parts.

\subsubsection{Void volume fraction}

The void volume fractions $\left(\rho_{x}\right)$ obtained by VOLCO-X simulations are compared to experimental ones in Fig 26 For $\bar{V}_{f}=16 \mathrm{~mm} / \mathrm{s}$, it can be seen that there is a good correlation between numerical and experimental void volume fractions. VOLCO-X was able to predict, with excellent agreement to the experimental data, the almost null void volume fraction for $E / \bar{E}=1.5$.

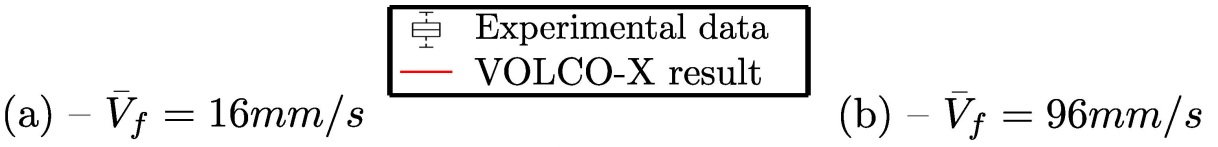
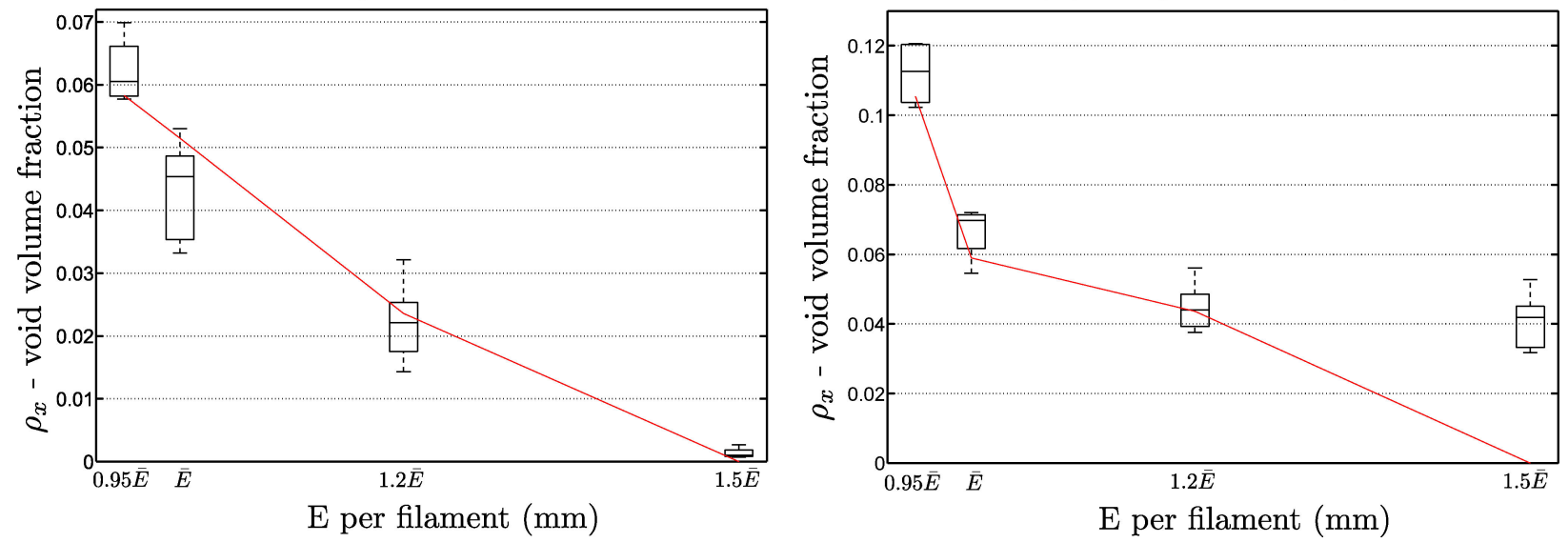

Figure 26: Experimental and numerical void volume fractions $\left(\rho_{x}\right)$ as function of $E$ for $\bar{V}_{f}=16 \mathrm{~mm} / \mathrm{s}$ and $\bar{V}_{f}=96 \mathrm{~mm} / \mathrm{s}$.

For $\bar{V}_{f}=96 \mathrm{~mm} / \mathrm{s}$, the void volume fraction calculated by VOLCO-X presented good correlation to experimental data for all $E$ values, except when $E / \bar{E}=1.5$. In this case, the numerical void volume fraction was $\rho_{x}=0.0$, while the experimental one was around $\rho_{x}=0.04$. The observed discrepancy in void volume fraction occurred because the experimental printed part had less material to fill the voids between deposited filaments than the numerical printed part, resulting in a greater experimental void volume fraction. 


\section{Applications and future work}

Based on the results achieved, some applications to VOLCO-X can be foreseen. It can be used in conjunction to optimisation methodologies to find optimal printing parameters. For instance, simulations could be used to help optimise printing parameters for printing structures that must be watertight for fluid applications, reducing voids within the printed part, or for 3D printed heat exchange units, to predict contact area and/or porosity for thermal conduction. Another possible application is predicting electrical conductivity of 3D printed conductive filaments. The method may also be used in slicers to predict under or over-extrusion and allow for optimisation or virtual inspection of predicted errors/faults before manufacture. Furthermore, VOLCO-X results can be used to predict equivalent structural properties of 3D printed parts with the aid of micromechanical modelling [50 54].

Nevertheless, the simulation approach behind VOLCO-X is flexible enough to include further developments not implemented in the present version, such as thermal effects, multi-material 3D printing (e.g. by having two separate phases in the modelling environment, that may take into account relative differences in material flow), effects from gravity and random 3D printing defects. 3D printing with orthogonal and multi-oriented layers as well as curvilinear printing paths can be investigated in deep in view of the here demonstrated capabilities. The prediction of 3D printed parts architectures combining several microstructures including full and partial infills (like scaffolds [32, 55], supports [56 58] and others) are among possibilities as well.

\section{Conclusion}

This work presents a new version of VOLCO, called VOLCO-X, in which a mathematical modelling approach was developed to take into account changes in material distribution when neighboring filaments are in contact. Moreover, the effects of the printing speed on the material distribution were also implemented in VOLCO-X. The results obtained, in fast computation times, consider a wide range of printing conditions, from fully dense structures to under-extruded structures with gaps. This simulation software can be used as a numerical tool in the design of functional printed parts, helping to predict the printed part dimensions, to choose adequate printing speed, to predict the void shape and material distribution, to calculate void volume fraction and to predict whether there will be gaps or defects within a printed part.

Experiments were performed to validate the new proposed numerical model. In the first set of experiments, ABS-MG94 specimens were manufactured with different printing speeds, and it was observed that 
more material was deposited at the initial and final portions of the specimens printed with the highest printing speed. Therefore, a higher void volume fraction was observed in the central portion of these specimens. These phenomena were capture successfully with VOLCO-X.

In the second set of experiments, the distance between centres of deposited filament $(d)$ was varied. VOLCO-X was able to predict the tendencies in the shape of the voids: for small $d$ values, triangles were observed in the microstructure, while diamond voids were observed when $d$ increased.

In the third set of experiments, the amount of material deposited per filament was varied, which is represented by the variable $E$. In these experiments, VOLCO-X could also predict successfully the trends in void shape observed in the experiments: for small $E$ values, diamond shapes were seen, and the voids shrunk with the increase in $E$.

From the overall results, it can be observed that VOLCO-X was able to predict final dimensions of printed parts with good agreement to the dimensions measured experimentally. Moreover, VOLCO-X was able to predict onset of gap faults within the microstructure. VOLCO-X can, hence, be confidently used to determine printing parameters that generate printed parts with structural integrity (without gap faults). The simulations were able to predict the void volume fraction at the central cross section of the specimens with good agreement to experimental data.

The mentioned applications and possible extensions depict the importance of a computationally efficient numerical method that correctly predicts the material distribution of FFF printed, as VOLCO-X does. There is no other model capable of predicting, in fast computation time, the material distribution as a function of printing speed and distance between deposited filaments.

\section{Acknowledgements}

This work was supported by the São Paulo Research Foundation (FAPESP) [grant numbers 2016/178356, 2017/22123-8 and 2017/09419-5], the Coordenação de Aperfeiçoamento de Pessoal de Nível Superior (CAPES), Conselho Nacional de Desenvolvimento Científico e Tecnológico (CNPq) [grant number 407754/20180] and Engineering and Physical Sciences Research Council [grant number EP/P027261/1].

\section{Declaration of interest}

None. 


\section{References}

1. Wickramasinghe S, Do T, Tran P. FDM-based 3D printing of polymer and associated composite: A review on mechanical properties, defects and treatments. Polymers 2020;12(7). doi 10.3390/polym12071529

2. Jones R, Haufe P, Sells E, Iravani P, Olliver V, Palmer C, Bowyer A. Reprap-the replicating rapid prototyper. Robotica 2011;29(1):177-91. doi 10.1017/S026357471000069X

3. Kroll E, Artzi D. Enhancing aerospace engineering students' learning with 3D printing wind-tunnel models. Rapid Prototyping Journal 2011;17(5):393-402. doi 10.1108/13552541111156522

4. Quinn M. Fused filament fabrication of polycarbonate components in a simulated on-orbit environment. Master's thesis; Delft University of Technology; 2018.

5. Teo Z, New T, Li S, Pfeiffer T, Nagel B, Gollnick V. Wind tunnel testing of additive manufactured aircraft components. Rapid Prototyping Journal 2018;24(5):886-93. doi 10.1108/RPJ-06-2016-0103

6. Love LJ, Noakes MW, Post BK, Rhyne BJ, Gaul KT. Feasibility of using additive manufacturing to produce automotive tooling. Tech. Rep.; Oak Ridge National Lab.(ORNL), Oak Ridge, TN (United States); 2018. doi 10.2172/1463997.

7. Leal R, Barreiros F, Alves L, Romeiro F, Vasco J, Santos M, Marto C. Additive manufacturing tooling for the automotive industry. The International Journal of Advanced Manufacturing Technology 2017;92(5-8):1671-6. doi 10.1007/ s00170-017-0239-8

8. Singh S, Ramakrishna S, Singh R. Material issues in additive manufacturing: A review. Journal of Manufacturing Processes 2017;25:185-200. doi 10.1016/j.jmapro.2016.11.006

9. Norman J, Madurawe RD, Moore CM, Khan MA, Khairuzzaman A. A new chapter in pharmaceutical manufacturing: 3D-printed drug products. Advanced Drug Delivery Reviews 2017;108:39-50. doi 10.1016/j.addr.2016.03.001

10. Katkar RA, Taft RM, Grant GT. 3D volume rendering and 3D printing (additive manufacturing). Dental Clinics of North America 2018;62(3):393-402. doi 10.1016/j.cden.2018.03.003

11. Guo N, Leu MC. Additive manufacturing: technology, applications and research needs. Frontiers of Mechanical Engineering 2013;8(3):215-43. doi 10.1007/s11465-013-0248-8

12. Lipton JI, Cutler M, Nigl F, Cohen D, Lipson H. Additive manufacturing for the food industry. Trends in food science \& technology 2015;43(1):114-23. doi 10.1016/j.tifs.2015.02.004

13. Brenken B, Barocio E, Favaloro A, Kunc V, Pipes RB. Fused filament fabrication of fiber-reinforced polymers: A review. Additive Manufacturing 2018; doi 10.1016/j.addma.2018.01.002

14. Gao W, Zhang Y, Ramanujan D, Ramani K, Chen Y, Williams CB, Wang CC, Shin YC, Zhang S, Zavattieri PD. The status, challenges, and future of additive manufacturing in engineering. Computer-Aided Design 2015;69:65-89. doi $10.1016 /$ j.cad.2015.04.001

15. de Macedo RQ, Ferreira RTL, Jayachandran KP. Determination of mechanical properties of FFF 3D printed material by assessing void volume fraction, cooling rate and residual thermal stresses. Rapid Prototyping Journal 2019;25(10):1661-83. doi 10.1108/RPJ-08-2018-0192

16. Gibson I, Rosen D, Stucker B, Khorasani M. Additive Manufacturing Technologies. Springer International; 2021. doi 10. 1007/978-3-030-56127-7 3rd ed.

17. Ferreira RTL, Amatte IC, Dutra TA, Bürger D. Experimental characterization and micrography of 3D printed PLA and PLA reinforced with short carbon fibers. Composites Part B: Engineering 2017;124:88-100. doi 10.1016/j.compositesb. 2017.05 .013 
18. Ning F, Cong W, Hu Y, Wang H. Additive manufacturing of carbon fiber-reinforced plastic composites using fused deposition modeling: Effects of process parameters on tensile properties. Journal of Composite Materials 2017;51(4):45162. doi $10.1177 / 0021998316646169$

19. Wu W, Geng P, Li G, Zhao D, Zhang H, Zhao J. Influence of layer thickness and raster angle on the mechanical properties of 3D-printed PEEK and a comparative mechanical study between PEEK and ABS. Materials 2015;8(9):583446. doi $10.3390 / \mathrm{ma} 8095271$

20. Dutra TA, Ferreira RTL, Resende HB, Guimarães A. Mechanical characterization and asymptotic homogenization of 3D-printed continuous carbon fiber-reinforced thermoplastic. Journal of the Brazilian Society of Mechanical Sciences and Engineering 2019;41(3):133. doi $10.1007 / \mathrm{s} 40430-019-1630-1$

21. van der Klift F, Koga Y, Todoroki A, Ueda M, Hirano Y, Matsuzaki R. 3D printing of continuous carbon fibre reinforced thermo-plastic (CFRTP) tensile test specimens. Open Journal of Composite Materials 2015;6(01):18. doi 10.4236/ojcm. 2016.61003

22. Dickson AN, Barry JN, McDonnell KA, Dowling DP. Fabrication of continuous carbon, glass and kevlar fibre reinforced polymer composites using additive manufacturing. Additive Manufacturing 2017;16:146-52. doi 10.1016/j.addma.2017. 06.004

23. Wei X, Li D, Jiang W, Gu Z, Wang X, Zhang Z, Sun Z. 3D printable graphene composite. Scientific reports 2015;5:11181. doi $10.1038 /$ srep11181

24. Espalin D, Alberto Ramirez J, Medina F, Wicker R. Multi-material, multi-technology FDM: exploring build process variations. Rapid Prototyping Journal 2014;20(3):236-44. doi 10.1108/RPJ-12-2012-0112

25. Rodríguez JF, Thomas JP, Renaud JE. Mechanical behavior of acrylonitrile butadiene styrene fused deposition materials modeling. Rapid Prototyping Journal 2003;9(4):219-30. doi 10.1108/13552540310489604

26. Liu X, Shapiro V. Homogenization of material properties in additively manufactured structures. Computer-Aided Design 2016;78:71-82. doi $10.1016 / \mathrm{j} . \mathrm{cad} .2016 .05 .017$.

27. Borille AV, Gomes JO, Lopes D. Geometrical analysis and tensile behaviour of parts manufactured with flame retardant polymers by additive manufacturing. Rapid Prototyping Journal 2017;23(1):169-80. doi 10.1108/RPJ-09-2015-0130

28. Somireddy M, Czekanski A, Singh CV. Development of constitutive material model of 3D printed structure via FDM. Materials Today Communications 2018;15:143-52. doi 10.1016/j.mtcomm.2018.03.004

29. Miller AT. Fatigue and cyclic loading of 3D printed soft polymers for orthopedic applications. Ph.D. thesis; Georgia Institute of Technology; 2017.

30. Wang L, Gramlich WM, Gardner DJ. Improving the impact strength of polylactic acid(PLA) in fused layer modeling (FLM). Polymer 2017;114:242-8. doi 10.1016/j.polymer.2017.03.011

31. Bellehumeur C, Li L, Sun Q, Gu P. Modeling of bond formation between polymer filaments in the fused deposition modeling process. Journal of Manufacturing Processes 2004;6(2):170-8. doi 10.1016/S1526-6125(04)70071-7.

32. Gleadall A, Ashcroft I, Segal J. VOLCO: A predictive model for 3D printed microarchitecture. Additive Manufacturing 2018;21:605-18. doi $10.1016 / \mathrm{j}$.addma.2018.04.004

33. Kuznetsov VE, Solonin AN, Urzhumtsev OD, Schilling R, Tavitov AG. Strength of PLA components fabricated with fused deposition technology using a desktop 3D printer as a function of geometrical parameters of the process. Polymers $2018 ; 10(3): 313$.

34. Kalita SJ, Bose S, Hosick HL, Bandyopadhyay A. Development of controlled porosity polymer-ceramic composite scaffolds 
via fused deposition modeling. Materials Science and Engineering: C 2003;23(5):611-20. doi 10.1016/S0928-4931(03) 00052-3

35. Sun Q, Rizvi G, Bellehumeur C, Gu P. Effect of processing conditions on the bonding quality of FDM polymer filaments. Rapid Prototyping Journal 2008;14(2):72-80. doi 10.1108/13552540810862028

36. Gurrala PK, Regalla SP. Part strength evolution with bonding between filaments in fused deposition modelling: This paper studies how coalescence of filaments contributes to the strength of final FDM part. Virtual and Physical Prototyping 2014;9(3):141-9. doi 10.1080/17452759.2014.913400.

37. Pokluda O, Bellehumeur CT, Vlachopoulos J. Modification of frenkel's model for sintering. AIChE journal 1997;43(12):3253-6. doi 10.1002/aic.690431213

38. Zhang W, Wu AS, Sun J, Quan Z, Gu B, Sun B, Cotton C, Heider D, Chou TW. Characterization of residual stress and deformation in additively manufactured ABS polymer and composite specimens. Composites Science and Technology $2017 ; 150: 102-10$.

39. Somireddy M, Czekanski A. Mechanical characterization of additively manufactured parts by FE modeling of mesostructure. Journal of Manufacturing and Materials Processing 2017;1(2):18. doi 10.3390/jmmp1020018

40. Rodriguez JF, Thomas JP, Renaud JE. Characterization of the mesostructure of fused-deposition acrylonitrile-butadienestyrene materials. Rapid Prototyping Journal 2000;6(3):175-86. doi 10.1108/13552540010337056

41. Comminal R, Serdeczny MP, Pedersen DB, Spangenberg J. Numerical modeling of the strand deposition flow in extrusionbased additive manufacturing. Additive Manufacturing 2018;20:68 - 76. doi 10.1016/j.addma.2017.12.013

42. Comminal R, Serdeczny MP, Pedersen DB, Spangenberg J. Motion planning and numerical simulation of material deposition at corners in extrusion additive manufacturing. Additive Manufacturing 2019;29:100753. doi 10.1016/j.addma. 2019.06 .005

43. Serdeczny MP, Comminal R, Pedersen DB, Spangenberg J. Numerical simulations of the mesostructure formation in material extrusion additive manufacturing. Additive Manufacturing 2019;28:419 -29. doi 10.1016/j .addma.2019.05.024

44. Zhang Y, Chou K. A parametric study of part distortions in fused deposition modelling using three-dimensional finite element analysis. Proceedings of the Institution of Mechanical Engineers, Part B: Journal of Engineering Manufacture 2008;222(8):959-68. doi 10.1243/09544054JEM990.

45. Graybill B. Development of a predictive model for the design of parts fabricated by fused deposition modeling. Ph.D. thesis; University of Missouri-Columbia; 2010.

46. Bellini A. Fused deposition of ceramics: a comprehensive experimental, analytical and computational study of material behavior, fabrication process and equipment design. Ph.D. thesis; Drexel University; 2002.

47. Park SI, Rosen DW, Choi Sk, Duty CE. Effective mechanical properties of lattice material fabricated by material extrusion additive manufacturing. Additive Manufacturing 2014;1:12-23. doi 10.1016/j.addma.2014.07.002

48. Qi X, Chen G, Li Y, Cheng X, Li C. Applying neural-network-based machine learning to additive manufacturing: Current applications, challenges, and future perspectives. Engineering 2019;5(4):721 -9. doi 10.1016/j.eng.2019.04.012

49. Wang C, Tan X, Tor S, Lim C. Machine learning in additive manufacturing: State-of-the-art and perspectives. Additive Manufacturing 2020;36:101538. doi $10.1016 / j$.addma.2020.101538

50. de Macedo RQ, Ferreira RTL, Guedes JM, Donadon MV. Intraply failure criterion for unidirectional fiber reinforced composites by means of asymptotic homogenization. Composite Structures 2017;159:335-49. doi 10.1016/j.compstruct. 2016.08 .027 
51. de Macedo RQ, Ferreira RTL, Donadon MV, Guedes JM. Elastic properties of unidirectional fiber-reinforced composites using asymptotic homogenization techniques. Journal of the Brazilian Society of Mechanical Sciences and Engineering 2018;40(5):255. doi $10.1007 / \mathrm{s} 40430-018-1174-9$

52. Ferreira RT, Rodrigues HC, Guedes JM, Hernandes JA. Hierarchical optimization of laminated fiber reinforced composites. Composite Structures 2014;107:246-59. doi 10.1016/j.compstruct.2013.07.051

53. Hierarchical optimization of fiber reinforced composites for natural frequencies. Engineering Optimization IV - Proceedings of the 4th International Conference on Engineering Optimization, ENGOPT; 2014. doi 10.1201/b17488-173

54. Dutra TA, Ferreira RT, Resende HB, Guimarães A, Guedes JM. A complete implementation methodology for asymptotic homogenization using a finite element commercial software: preprocessing and postprocessing. Composite Structures 2020;245(10):112305. doi $10.1016 / \mathrm{j}$. compstruct.2020.112305

55. Castro APG, Ruben RB, Gonçalves SB, Pinheiro J, Guedes JM, Fernandes PR. Numerical and experimental evaluation of tpms gyroid scaffolds for bone tissue engineering. Computer Methods in Biomechanics and Biomedical Engineering 2019;22(6):567-73. doi 10.1080/10255842.2019.1569638

56. Strano G, Hao L, Everson RM, Evans KE. A new approach to the design and optimisation of support structures in additive manufacturing. Int J Adv Manuf Technol 2013;66:1247-1254. doi 10.1007/s00170-012-4403-x

57. Jiang J, Xu X, Stringer J. Support structures for additive manufacturing: A review. Journal of Manufacturing and Materials Processing 2018;2(4). doi 10.3390/jmmp2040064

58. Jang S, Moon B, Lee K. Free-floating support structure generation. Computer-Aided Design 2020;128:102908. doi 10. $1016 / j \cdot c a d .2020 .102908$ 